\title{
Performance Analysis of Two-Way Relay NOMA Systems with Hardware Impair- ments and Channel Estimation Errors
}

\author{
Xinji Tian ${ }^{1}$, Qianqian $\mathrm{Li}^{1}$, Xingwang $\mathrm{Li}^{1}$, Hui Zhang ${ }^{2 *}$, Khaled Rabie ${ }^{3}$ \\ and Charles Casimiro Cavalcante ${ }^{4}$ \\ ${ }^{1}$ School of Physics and Electronic Information Engineering, Henan Polytechnic University, Jiaozuo, 454000, \\ China \\ [e-mail: tian215216@sohu.com, liqianqian_lf@163.com, lixingwang@hpu.edu.cn] \\ ${ }^{2}$ School of Energy Science and Engineering, Henan Polytechnic University, Jiaozuo, 454000, China \\ [e-mail: caikuangzhang@163.com] \\ ${ }^{3}$ School of Electrical Engineering, Manchester Metropolitan University, Manchester M1 7JW, K. \\ [e-mail:k.rabie@mmu.ac.uk] \\ ${ }^{4}$ Wireless Telecommunications Research Group, Federal University of Ceará, Campus do Pici, Bl. 722, ZIP \\ 60455-760, Fortaleza-CE, Brazil \\ [e-mail: charles@gtel.ufc.br] \\ *Corresponding author: Hui Zhang
}

Received March 8, 2019; revised May 4, 2019; accepted May 16, 2019;

published November 30, 2019

\begin{abstract}
In this paper, we consider a two-way relay non-orthogonal multiple access (TWR-NOMA) system with residual hardware impairments (RHIs) and channel estimation errors (CEEs), where two group users exchange their information via the decode-and-forward (DF) relay by using NOMA protocol. To evaluate the performance of the considered system, exact analytical expressions for the outage probability of the two groups users are derived in closed-form. Moreover, the asymptotic outage behavior in the high signal-to-noise ratio (SNR) regime is examined and the diversity order is derived and discussed. Numerical simulation results verify the accuracy of theoretical analyses, and show that: $i$ ) RHIs and CEEs have a deleterious effects on the outage probabilities; ii) CEEs have significant effects on the performance of the near user; iii) Due to the RHIs, CEEs, inter-group interference and intra-group interference, there exists error floors for the outage probability.
\end{abstract}

Keywords: Non-orthogonal multiple access, residual hardware impairments, two-way relay, channel estimation errors.

This work was supported in part by the Henan Scientific and Technological Research Project (182102210307), in part by the Doctoral Scientific Funds of Henan polytechnic University (no. B2016-34), in part by the Fundamental Research Funds for the Universities of Henan Province (NSFRF180309), in part by the Outstanding Youth Science Foundation of Henan Polytechnic University (J2019-4). 


\section{Introduction}

$\mathbf{N}_{\text {on-orthogonal multiple access (NOMA) has been identified as a promising technique for }}$ the fifth generation (5G) mobile communication network since it has the advantages of low latency, massive connectivity and high spectral efficiency [1-3]. In general, NOMA can be classified into two categories: power domain NOMA [4] and code domain NOMA [5]. For power domain NOMA, multi-users can be simultaneously served by the same base station using the same resource, while for code domain NOMA, the signals of different users are spread by using different codes and then multiplexed over the same time-frequency resources [6]. In this paper, we consider the power domain NOMA. It is worth noting that the power domain NOMA mentioned through this paper will be replaced by NOMA. For NOMA systems, in order to ensure a trade-off between throughput and user fairness, more powers are allocated to the users with poorer channel conditions. At the transmitter, the superposition signals are sent by power multiplexing, while the signals can be separately decoded at receivers by successive interference cancellation (SIC) [7].

Cooperative communication is another effective way to improve spectral efficiency, reduce transmit power and broaden the network coverage [8]. To further improve the system performance, two-way relay (TWR) was proposed for its ability to exchange information with the aid of a common relay with bidirectional information-transmission [9]. For combat channel fading and improve transmission reliability, the diversity behavior of the generalized MIMO TWR networks was studied in [10], where a two relay antenna selection (RAS) scheme was proposed. In [11], the outage behavior of a TWR network subject to a nonlinear transmission at the relay was studied, where fixed-gain and variable-gain amplify-and-forward (AF) relay were taken into account. Considering mixed asymmetric line-of-sight (LoS)/non-LoS (NLoS) fading scenario, the authors in [12] investigated the outage probability and channel capacity of the TWR networks. In [13], a distributed robust beamforming scheme was designed to minimize the total transmit power of the cognitive TWR networks. To further enhance the performance, the multi-antenna technique was introduced to the TWR networks [14], in which an optimal linear beamforming scheme was proposed to minimize the weighted mean squared error. As a further advance, massive multiple-input multiple-output (MIMO) inspired TWR network were involved in [15], where the impact of residual hardware impairments (RHIs) was investigated by considering Rician fading channels.

Recently, the combination of TWR and NOMA, known as TWR-NOMA, has drawn a considerable research attention, since it exploits advantages of both TWR and NOMA [16-19]. In [16], the outage probability and ergodic rates of a TWR-NOMA system were investigated, in which perfect and imperfect SIC were analyzed. Sparked by full-duplex, the authors in [17] proposed a full duplex cooperative NOMA system, the outage probability and ergodic capacity of the proposed system were derived. For increase the system throughput and reduce the signaling overhead, the multi-pair TWR-NOMA network were developed in [18], in which an optimal group decoding scheduling scheme joint fair rate allocation was proposed for uplink and downlink. With the emphasis on secure transmission, different decoding schemes of the NOMA-based full-duplex TWR networks were proposed in [19] based on SIC for the legitimate users, relay and eavesdroppers.

Although the above-mentioned works provide a solid foundation on the TWR-NOMA systems, one of the limitations of the above works is that the perfect hardware is assumed. In practice, due to deploying low-cost and low power efficiency RF components, the transceivers 
are prone to hardware imperfections and impaired by some types of hardware impairments, such as in-phase/quadrature-phase (I/Q) imbalance, amplifier non-linearities, and phase noise [20-22]. Although some signal processing algorithms can be used to compensate for the above imperfections, there still remains some RHIs due to estimation errors, inaccurate calibration and different types of noise [23], [24]. As stated in [20] [22] [25], the RHIs have significant effects on the system performance. The authors in [20] quantified the aggregated impact of RHIs on dual-hop relaying systems. The joint impact of RHIs and imperfect CSI on the multi-relay NOMA system was investigated in [22]. The authors in [25] derived the expressions of outage probability and approximate ergodic sum rate for analysis the deleterious effect of RHIs on the dual-hop NOMA network. The performance of wireless communication systems in the presence of RHIs has been extensively studied, e.g., see [26-28] and the references therein. The authors in [26] analyzed the effect of RHIs on the ergodic channel and ergodic sum rates of optimal and linear minimum mean-square-error (MMSE) receivers of MIMO systems. In [27], authors investigated the lower bound for the achievable sum rate of regular and large-scale MIMO systems with zero-forcing receivers in the presence of RHIs. In [28], exact closed-form expressions of outage probability and asymptotic expressions were derived in a TWR cooperative network with opportunistic relay selection, and the allocation of the fixed hardware impairments are analyzed as well. Recently, there are some research works dealing with the performance analysis of related topics of NOMA with RHIs, e.g., [29-31]. In [29], the authors investigated the performance of the one way cooperative NOMA network with RHIs by deriving a closed-form expression for the outage probability. Considering RHIs at the relay, [30] derived analytical expressions for the outage probability and symbol error rate of a TWR network. In [31], a one way relay dual-hop NOMA network with RHIs at source, relay and destination was considered, in which the outage probability, asymptotic individual ergodic rate (IER) and ergodic sum rate (ESR) were obtained. Nevertheless, the above works assume that all nodes have perfect channel state information (CSI). In fact, the performance of wireless communication system is greatly affected by wireless channel, such as shadow fading and frequency selective fading, which makes the path between transmitter and receiver very complicated. Due to randomness nature of wireless channels, it is a great challenge to obtain perfect channel knowledge. The common way of doing this is to use some estimation algorithms to obtain the estimated. However, the perfect estimation is not available due to channel estimation errors (CEEs). Therefore, it is of significance to investigate the joint impact of RHIs and CEEs on the TWR-NOMA system.

Motivation and Contribution: Motivated by the above discussion, different from the existing works on TWR-NOMA systems, we investigate the performance of TWR-NOMA systems in the presence of RHIs and CCEs, where two groups NOMA users exchange their information with the aid of decode-and-forward (DF) relay. The contributions of this paper are summarized as follows:

- Contrary to the existing research works on the performance of TWR-NOMA, we consider two practical factors, namely, RHIS and CEE. We aim at investigating the joint effects of the two factors on TWR-NOMA networks, which is a valuable problem for practical system analysis and design.

- We derived exact closed-form analytical expressions for the outage probability of the far users and near users. In addition, we also derived exact analytical expressions for the outage probability of the considered network under the condition of ideal RF components and CSI.

- We examine the asymptotic outage behavior of the users and discuss the diversity order in the high signal-to-noise ratio (SNR) region. It reveals that RHIs and CEEs 
can cause the outage performance to deteriorate. Moreover, the results show that there exists an error floor for the outage probability due to inter/intra group interference and CEEs, and the diversity order is zero. Additionally, CEEs have a deleterious effect on the outage probability on the near users.

Organization: The rest of this paper is organized as follows. In section II, we present the TWR-NOMA system model in the presence of RHIs and CEEs. The exact closed-form analytical and asymptotic expressions for the outage probability and diversity order are derived and discussed in section III. In section IV, we present some numerical and simulation results to verify the accuracy of our analysis. A brief summary of this paper is concluded in section $\mathrm{V}$.

Notations: In this paper, the main notations are shown as follows: $E\{\}$ denotes the expectation operation, $\operatorname{Pr}(\cdot)$ is the probability, $\Pi(\cdot)$ denotes the continuous multiplication operation, $\sum(\cdot)$ is the summation.

\section{System Model}

A TWR-NOMA system is considered, which consists of one two-antenna relay $R$ and two groups of NOMA users $G_{1}=\left\{U_{1}, U_{2}\right\}$ and $G_{2}=\left\{U_{3}, U_{4}\right\}$ as illustrated in Fig. 1. For NOMA users, we assume that two types of users are deployed: $U_{1}$ and $U_{3}$ are the near users, and $U_{2}$ and $U_{4}$ are the far users. The users of same type aim to exchange their informations via a two-antenna relay $R$, namely $R_{1}$ and $R_{2}$. In addition, there is no direct link between two groups of users due to heavy shadowing. We also assume that all users are equipped with a single antenna and operate in a half-duplex mode. $h_{i}, i \in\{1,2,3,4\}$, denotes the channel fading coefficients between $U_{i}$ and $R$, where $h_{i}$ follows independent Rayleigh random variables with $\left|h_{i}\right|^{2} \sim C N\left(0, \delta_{i}^{2}\right)$.

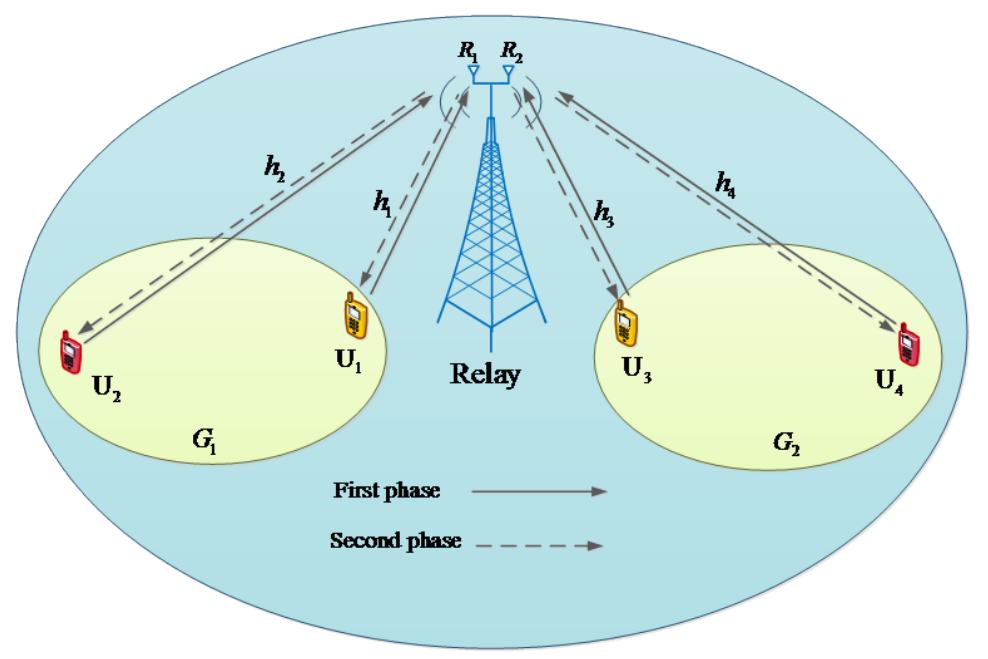

Fig. 1. System model 
In practice communication systems, it is a great challenge to obtain perfect CSI due to the CEEs. Thus, the channel fading coefficient can be modeled as $h_{i}=\hat{h}_{i}+e_{i}$, where $\hat{h}_{i}$ represent the estimated channels coefficient, $e_{i}$ are the CEEs, which can be modeled by Gaussian random variable with $e_{i} \sim C N\left(0, \delta_{e_{i}}^{2}\right)$ [32].

The whole communication is completed in two phases: 1) multiple access channel (MAC) phase; 2) broadcasting (BC) phase.

1) MAC Phase: In this phase, two pairs of users simultaneously transmit the respective information to the intended antenna of the relay, and the received information at $R_{1}\left(R_{2}\right)$ suffers from interference from the users of $G_{2}\left(G_{1}\right)$. Thus, the received signals at $R_{1}$ and $R_{2}$ are respectively given by

$$
\begin{aligned}
& y_{R_{1}}=h_{1} \sqrt{a_{1} P_{u}} x_{1}+h_{2} \sqrt{a_{2} P_{u}} x_{2}+\varpi_{1} I_{2}+\eta_{r}+n_{R_{1}} \\
& y_{R_{2}}=h_{3} \sqrt{a_{3} P_{u}} x_{3}+h_{4} \sqrt{a_{4} P_{u}} x_{4}+\varpi_{1} I_{1}+\eta_{r}+n_{R_{2}}
\end{aligned}
$$

where $x_{i}$ are the transmitted signals by the user $U_{i}$, with $E\left\{x_{i}\right\}=1, i \in\{1,2,3,4\} . P_{u}$ is the transmission power. $a_{1}, a_{2}, a_{3}$ and $a_{4}$ are the corresponding power allocation coefficients, satisfying $a_{1}>a_{2}, a_{1}+a_{2}=1$, and $a_{3}>a_{4}, a_{3}+a_{4}=1 ; I_{2}$ is the inter-group interference signal (IS) from $R_{2}$ with $I_{2}=h_{3} \sqrt{a_{3} P_{u}} x_{3}+h_{4} \sqrt{a_{4} P_{u}} x_{4} ; \varpi_{1} \in[0,1]$ is the impact level of inter-group IS at $R ; I_{1}$ is the inter-group IS from $R_{1}$ with $I_{1}=h_{1} \sqrt{a_{1} P_{u}} x_{1}+h_{2} \sqrt{a_{2} P_{u}} x_{2} ; n_{R_{1}}$ and $n_{R_{2}}$ are additive white Gaussian noise (AWGN) with zero mean and $N_{0}$ variance at $R_{1}$ and $R_{2}$, respectively; $\eta_{r}$ represents the received RHIs at relay with $\eta_{r} \sim C N\left(0, \kappa_{r}^{2} P_{u} \sum_{i=1}^{4}\left|h_{i}\right|^{2}\right), \kappa_{r}$ is used to characterize the aggregated level of RHIs from the relay [33].

According to the DF protocol of NOMA, $R_{1}$ first decodes $x_{1}$ by treating signals from the users of another group as inter-group IS. Then, the received signal-to-interference plus noise rates (SINR) at $R_{1}$ to detect $x_{1}$ is given by

$$
\gamma_{R \rightarrow x_{1}}=\frac{\rho_{u}\left|\hat{h}_{1}\right|^{2} a_{1}}{\rho_{u}\left|\hat{h}_{2}\right|^{2} a_{2}+\sum_{k=1}^{4} \rho_{u}\left|\hat{h}_{k}\right|^{2} \kappa_{r}^{2}+\sum_{j=3}^{4} \rho_{u}\left|\hat{h}_{j}\right|^{2} \varpi_{1} a_{j}+\vartheta_{1}}
$$

where $\vartheta_{1}=\sum_{i=1}^{2} \rho_{u} \delta_{e_{i}}^{2} a_{i}+\sum_{j=3}^{4} \varpi_{1} \rho_{u} \delta_{e_{j}}^{2} a_{j}+\sum_{k=1}^{4} \kappa_{r}^{2} \rho_{u} \delta_{e_{1}}^{2}+1$, and $\rho_{u}=P_{u} / N_{0}$ denotes the transmit SNR at the user nodes.

Then, the SINR at relay to decode $x_{2}$ is given

$$
\gamma_{R \rightarrow x_{2}}=\frac{\rho_{u}\left|\hat{h}_{2}\right|^{2} a_{2}}{\sum_{k=1}^{4} \rho_{u}\left|\hat{h}_{k}\right|^{2} \kappa_{r}^{2}+\sum_{j=3}^{4} \rho_{u}\left|\hat{h}_{j}\right|^{2} \varpi_{1} a_{j}+\vartheta_{1}}
$$

2) BC Phase: In this phase, the relay decodes and forwards the received signals to all users. More specifically, the antennas $R_{2}$ and $R_{1}$ send the superposed signals $\left(\sqrt{b_{1} P_{r}} x_{1}+\sqrt{b_{2} P_{r}} x_{2}\right)$ 
and $\left(\sqrt{b_{3} P_{r}} x_{3}+\sqrt{b_{4} P_{r}} x_{4}\right)$ to $G_{2}$ and $G_{1}$, respectively, where $P_{r}$ is the transmission power at the relay, $b_{1}, b_{2}, b_{3}$ and $b_{4}$ are the power allocation coefficients. According the NOMA protocol, the far users are allocated more power than the near users $b_{2}>b_{1}$, with $b_{1}+b_{2}=1$ and $b_{4}>b_{3}$, with $b_{3}+b_{4}=1$.

The received signals at $U_{3}$ and $U_{4}$ denoted by $y_{U_{3}}$ and $y_{U_{4}}$, can be respectively expressed as

$$
\begin{aligned}
& y_{U_{3}}=h_{3}\left(\sum_{i=1}^{2} \sqrt{b_{i} P_{r}} x_{i}+\eta_{t}\right)+\varpi_{2} h_{3} \sum_{j=3}^{4} \sqrt{b_{j} P_{r}} x_{j}+n_{3} \\
& y_{U_{4}}=h_{4}\left(\sum_{i=1}^{2} \sqrt{b_{i} P_{r}} x_{i}+\eta_{t}\right)+\varpi_{2} h_{4} \sum_{j=3}^{4} \sqrt{b_{j} P_{r}} x_{j}+n_{4}
\end{aligned}
$$

where $\varpi_{2} \in[0,1]$ is the impact level of inter-group IS at the user nodes, $n_{3}$ and $n_{4}$ are AWGN with mean power $N_{0}$ at $U_{3}$ and $U_{4}$, respectively; $\eta_{t}$ represents the RHIs at the relay transmitter node, $\eta_{t} \sim C N\left(0, \kappa_{t}^{2} P_{r}\right), \kappa_{t}$ is used to characterize the aggregate level of impairments in the transmitter hardware.

We assume that the signals $x_{2}$ from the users can be correctly decoded at $R$ in the MAC phase. $U_{3}$ decodes the desired signal $x_{1}$ after using SIC to decode and eliminate $x_{2}$. The effective SINR at $U_{3}$ for the detection of $x_{2}$ is given by

$$
\gamma_{U_{3} \rightarrow x_{2}}=\frac{\rho_{r}\left|\hat{h}_{3}\right|^{2} b_{2}}{\rho_{r}\left|\hat{h}_{3}\right|^{2} b_{1}+\rho_{r}\left|\hat{h}_{3}\right|^{2} \kappa_{t}^{2}+\varpi_{2} \rho_{r}\left|\hat{h}_{3}\right|^{2}+\vartheta_{2}}
$$

where $\vartheta_{2}=\rho_{r} \delta_{e_{3}}^{2}\left(1+\kappa_{t}^{2}+\varpi_{2}\right)+1, \rho_{r}=P_{r} / N_{0}$ is the transmit SNR at the relay node.

Similarly, we assume that the signals $x_{1}$ from the users can be correctly decoded at $R$ in the MAC phase. After SIC operations, the received SINR at $U_{3}$ to decode $x_{1}$ is given by

$$
\gamma_{U_{3} \rightarrow x_{1}}=\frac{\rho_{r}\left|\hat{h}_{3}\right|^{2} b_{1}}{\rho_{r}\left|\hat{h}_{3}\right|^{2} \kappa_{t}^{2}+\varpi_{2} \rho_{r}\left|\hat{h}_{3}\right|^{2}+\vartheta_{2}}
$$

Then, the received SINR at $U_{4}$ to decode signal $x_{2}$ is given by

$$
\gamma_{U_{4} \rightarrow x_{2}}=\frac{\rho_{r}\left|\hat{h}_{4}\right|^{2} b_{2}}{\rho_{r}\left|\hat{h}_{4}\right|^{2} b_{1}+\rho_{r}\left|\hat{h}_{4}\right|^{2} \kappa_{t}^{2}+\varpi_{2} \rho_{r}\left|\hat{h}_{4}\right|^{2}+\vartheta_{3}}
$$

where $\vartheta_{3}=\rho_{r} \delta_{e_{4}}^{2}\left(1+\kappa_{t}^{2}+\varpi_{2}\right)+1$. After these processes, the information is exchanged between the NOMA users in $G_{1}$ and $G_{2}$.

\section{Outage Performance Analysis}

In this section, we first derive the exact analytical expressions for the outage probability. Then the asymptotic outage behavior at high SNR region is analyzed, and the diversity orders are also discussed. 


\subsection{Exact Outage Probability}

\section{1) Outage probability for $U_{1}$}

In the TWR-NOMA, the outage event of $U_{1}$ occurs in the following three cases: $i$ ) The information $x_{1}$ cannot be decoded by $R_{1}$; ii) $U_{3}$ cannot decode $x_{2}$ successfully; iii) The information $x_{1}$ cannot be decoded by $U_{3}$, while $U_{3}$ can first decode the information $x_{2}$ correctly. Hence, the outage probability of $U_{1}$ can be written as

$$
\mathrm{P}_{U_{1}}^{\text {out }}=1-\operatorname{Pr}\left(\gamma_{R \rightarrow x_{1}}>\gamma_{\text {thf }}\right) \operatorname{Pr}\left(\gamma_{U_{3} \rightarrow x_{2}}>\gamma_{\text {thm }}, \gamma_{U_{3} \rightarrow x_{1}}>\gamma_{\text {thf }}\right)
$$

where $\gamma_{\text {thf }}=2^{2 R_{f}}-1, \gamma_{\text {thm }}=2^{2 R_{m}}-1 . R_{f}$ and $R_{m}$ are the target rates at $U_{3}$ to detect $x_{1}$ and $x_{2}$, respectively.

Then, the outage probability of $U_{1}$ for the TWR-NOMA with RHIs and CEEs is provided in the following theorem.

Theorem 1. For the non-ideal cases $\left(\delta_{e_{i}}^{2} \neq 0, \kappa_{l} \neq 0, i \in\{1,2,3,4\}, l \in\{t, r\}\right)$, the closed-form expression for the outage probability of $U_{1}$ is expressed as

$$
\mathrm{P}_{U_{1}}^{\text {ni,out }}=1-e^{-\frac{\tau_{0}}{\hat{\delta}_{3}^{2}} \frac{\theta_{1} \vartheta_{1}}{\hat{\delta}_{1}^{2}}} \prod_{i=1}^{3} \lambda_{i}\left(\frac{\Phi_{1} \hat{\delta}_{1}^{2}}{\lambda_{1} \hat{\delta}_{1}^{2}+\theta_{1}}-\frac{\Phi_{2} \hat{\delta}_{1}^{2}}{\lambda_{2} \hat{\delta}_{1}^{2}+\theta_{1}}+\frac{\Phi_{3} \hat{\delta}_{1}^{2}}{\lambda_{3} \hat{\delta}_{1}^{2}+\theta_{1}}\right)
$$

where $\Phi_{1}=\left(\lambda_{2}-\lambda_{1}\right)^{-1}\left(\lambda_{3}-\lambda_{1}\right)^{-1}, \Phi_{2}=\left(\lambda_{3}-\lambda_{2}\right)^{-1}\left(\lambda_{2}-\lambda_{1}\right)^{-1}, \Phi_{3}=\left(\lambda_{3}-\lambda_{1}\right)^{-1}\left(\lambda_{3}-\lambda_{2}\right)^{-1}$, $\theta_{1}=\frac{\gamma_{\text {thf }}}{\rho_{u}\left(a_{1}-\gamma_{\text {thf }} \kappa_{r}^{2}\right)}$ with $a_{1}>\gamma_{\text {thf }} \kappa_{r}^{2}, \tau_{0}=\max \left(\tau_{2}, \tau_{1}\right)$, where $\tau_{1}=\frac{\gamma_{\text {thf }} \vartheta_{2}}{\rho_{r}\left(b_{1}-\gamma_{\text {thf }}\left(\kappa_{t}^{2}+\varpi_{2}\right)\right)}$ with $b_{1}>\gamma_{\text {thf }}\left(\kappa_{t}^{2}+\varpi_{2}\right), \quad \tau_{2}=\frac{\gamma_{t h m} \vartheta_{2}}{\rho_{r}\left(b_{2}-\gamma_{t h m}\left(b_{1}+\kappa_{t}^{2}+\varpi_{2}\right)\right)}$ with $b_{2}>\gamma_{t h m}\left(b_{1}+\kappa_{t}^{2}+\varpi_{2}\right)$, $\lambda_{1}=1 /\left(\rho_{u} \hat{\delta}_{2}^{2}\left(a_{2}+\kappa_{r}^{2}\right)\right), \lambda_{2}=1 /\left(\rho_{u} \hat{\delta}_{3}^{2}\left(\varpi_{1} a_{3}+\kappa_{r}^{2}\right)\right)$ and $\lambda_{3}=1 /\left(\rho_{u} \hat{\delta}_{4}^{2}\left(\varpi_{1} a_{4}+\kappa_{r}^{2}\right)\right)$.

Proof: See Appendix A.

Corollary 1. For the ideal cases $\left(\delta_{e_{i}}^{2}=0, \kappa_{l}=0\right)$, the analytical expression for the outage probability of $U_{1}$ is expressed as

$$
\mathrm{P}_{U_{1}}^{\text {id,out }}=1-e^{-\frac{\gamma_{\text {thf }}}{\xi_{1}}-\frac{\tau_{0}^{\prime}}{\delta_{3}^{2}}} \prod_{i=4}^{6} \lambda_{i}\left(\frac{\Psi_{1} \xi_{1}}{\xi_{1} \lambda_{4}+\gamma_{\text {thf }}}-\frac{\Psi_{2} \xi_{1}}{\xi_{1} \lambda_{5}+\gamma_{\text {thf }}}+\frac{\Psi_{3} \xi_{1}}{\xi_{1} \lambda_{6}+\gamma_{\text {thf }}}\right)
$$

where $\Psi_{1}=\left(\lambda_{5}-\lambda_{4}\right)^{-1}\left(\lambda_{6}-\lambda_{4}\right)^{-1}, \Psi_{2}=\left(\lambda_{6}-\lambda_{5}\right)^{-1}\left(\lambda_{5}-\lambda_{4}\right)^{-1}, \Psi_{3}=\left(\lambda_{6}-\lambda_{4}\right)^{-1}\left(\lambda_{6}-\lambda_{5}\right)^{-1}$, $\tau_{0}^{\prime}=\max \left(\tau_{2}^{\prime}, \tau_{1}^{\prime}\right), \tau_{1}^{\prime}=\frac{\gamma_{\text {thf }}}{\rho_{r}\left(b_{1}-\gamma_{t h f} \varpi_{2}\right)}$ with $b_{1}>\gamma_{t h f} \varpi_{2}$ and $\tau_{2}^{\prime}=\frac{\gamma_{t h m}}{\rho_{r}\left(b_{2}-\gamma_{t h m}\left(b_{1}+\varpi_{2}\right)\right)}$ with $b_{2}>\gamma_{\text {thm }}\left(b_{1}+\varpi_{2}\right) \quad, \quad \xi_{1}=\rho_{u} a_{1} \delta_{1}^{2} \quad, \quad \lambda_{4}=1 /\left(\rho_{u} a_{2} \delta_{2}^{2}\right) \quad, \quad \lambda_{5}=1 /\left(\varpi_{1} \rho_{u} a_{3} \delta_{3}^{2}\right) \quad$ and $\lambda_{6}=1 /\left(\varpi_{1} \rho_{u} a_{4} \delta_{4}^{2}\right)$. 
Proof: See Appendix B.

2) Outage probability for $U_{2}$

The outage events of $U_{2}$ include four conditions as follows: $i$ ) The information $x_{1}$ cannot be decoded correctly by relay. ii) The relay cannot decode $x_{2}$, while the relay can first decode $x_{1}$, successfully. iii) $U_{3}$ cannot decode $x_{2}$ correctly. iv) $x_{2}$ cannot be decoded by $U_{4}$ successfully. Therefore, the outage probability of $U_{2}$ can be expressed as

$$
P_{U_{2}}^{\text {out }}=1-\operatorname{Pr}\left(\gamma_{R \rightarrow x_{2}}>\gamma_{t h m}, \gamma_{R \rightarrow x_{1}}>\gamma_{\text {thf }}\right) \operatorname{Pr}\left(\gamma_{u_{3} \rightarrow x_{2}}>\gamma_{t h m}\right) \operatorname{Pr}\left(\gamma_{u_{4} \rightarrow x_{2}}>\gamma_{t h m}\right)
$$

Then, the outage probability of $U_{2}$ for the TWR-NOMA system with RHIs and CEEs is provided in the following theorem.

Theorem 2. For the non-ideal cases $\left(\delta_{e_{i}}^{2} \neq 0, \kappa_{l} \neq 0\right)$, the exact analytical expression of $U_{2}$ in terms of outage probability is expressed as

$$
\begin{aligned}
& \mathrm{P}_{U_{2}}^{\text {ni,out }}=1-e^{-\left(\frac{\theta_{2} \vartheta_{1}}{\delta_{2}^{2}}+\frac{\theta_{1} \vartheta_{1}}{\delta_{1}^{2}}+\frac{\tau_{2}}{\delta_{3}^{2}}+\frac{\tau_{3}}{\delta_{4}^{2}}\right)} \frac{\hat{\delta}_{2}^{2} \hat{\delta}_{1}^{2} \lambda_{1}^{\prime} \lambda_{2}^{\prime}}{\left(\theta_{2} \kappa_{r}^{2} \rho_{u} \hat{\delta}_{1}^{2}+\hat{\delta}_{2}^{2}\right)\left(\theta_{1} \rho_{u} \hat{\delta}_{2}^{2}\left(a_{2}+\kappa_{r}^{2}\right)+\hat{\delta}_{1}^{2}\right)\left(\lambda_{2}^{\prime}-\lambda_{1}^{\prime}\right)} \\
& \times\left(\frac{\hat{\delta}_{1}^{2} \hat{\delta}_{2}^{2}}{\theta_{2} \hat{\delta}_{1}^{2}+\theta_{1} \hat{\delta}_{2}^{2}+\lambda_{1}^{\prime} \hat{\delta}_{1}^{2} \hat{\delta}_{2}^{2}}-\frac{\hat{\delta}_{1}^{2} \hat{\delta}_{2}^{2}}{\theta_{2} \hat{\delta}_{1}^{2}+\theta_{1} \hat{\delta}_{2}^{2}+\lambda_{2}^{\prime} \hat{\delta}_{1}^{2} \hat{\delta}_{2}^{2}}\right)
\end{aligned}
$$

where $\lambda_{1}^{\prime}=1 /\left(\rho_{u} \hat{\delta}_{3}^{2}\left(\varpi_{1} a_{3}+\kappa_{r}^{2}\right)\right)$ and $\lambda_{2}^{\prime}=1 /\left(\rho_{u} \hat{\delta}_{4}^{2}\left(\varpi_{1} a_{4}+\kappa_{r}^{2}\right)\right) \cdot \theta_{2}=\frac{\gamma_{\text {thm }}}{\rho_{u}\left(a_{2}-\gamma_{t h m} \kappa_{r}^{2}\right)}$ with $a_{2}>\gamma_{t h m} \kappa_{r}^{2} . \tau_{3}=\frac{\gamma_{t h m} \vartheta_{3}}{\rho_{r}\left(b_{2}-\gamma_{t h m}\left(b_{1}+\kappa_{t}^{2}+\varpi_{2}\right)\right)}$ with $b_{2}>\gamma_{t h m}\left(b_{1}+\kappa_{t}^{2}+\varpi_{2}\right)$.

Proof: See Appendix C.

Corollary 2. For the ideal cases $\left(\delta_{e_{i}}^{2}=0, \kappa_{l}=0\right)$, the analytical expression for the outage probability with the ideal case of $U_{2}$ is expressed as

$$
\mathrm{P}_{U_{2}}^{\mathrm{id}, \text { out }}=1-e^{-\left(\frac{\gamma_{\text {thm }} \beta_{t}}{\rho_{u} a_{2}}+\frac{\gamma_{\text {thf }}}{\rho_{u} a_{1} \delta_{1}}+\frac{\tau_{2}^{\prime}}{\delta_{3}^{2}}+\frac{\tau_{2}^{\prime}}{\delta_{4}^{2}}\right)} \frac{\lambda_{5} \lambda_{6}}{\beta_{t} \delta_{2}^{2}\left(\lambda_{6}-\lambda_{5}\right)} \sum_{k=5}^{6} \frac{(-1)^{k+1} \rho_{u} a_{2}}{\beta_{t} \gamma_{t h m}+\lambda_{k} \rho_{u} a_{2}}
$$

where $\quad \tau_{2}^{\prime}=\frac{\gamma_{t h m}}{\rho_{r}\left(b_{2}-\gamma_{t h m}\left(b_{1}+\varpi_{2}\right)\right)} \quad$ with $\quad b_{2}>\gamma_{t h m}\left(b_{1}+\varpi_{2}\right) \quad$ and $\quad \beta_{t}=\frac{\gamma_{t h f} a_{2}}{a_{1} \delta_{1}^{2}}+\frac{1}{\delta_{2}^{2}}$. $Y^{\prime}=Y_{5}+Y_{6}$ with $\lambda_{5}=1 /\left(\varpi_{1} \rho_{u} a_{3} \delta_{3}^{2}\right), \lambda_{6}=1 /\left(\varpi_{1} \rho_{u} a_{4} \delta_{4}^{2}\right)$.

Proof: See Appendix D.

\subsection{Asymptotic Outage Probability}

To gain more insights, the asymptotic outage behavior is studied in the high SNR region. For the TWR-NOMA system, $\rho_{u}=\varepsilon \rho_{r} \rightarrow \infty$ and $\varepsilon>0$. The asymptotic SINR of (3) and (4) can be approximately expressed as 


$$
\begin{gathered}
\bar{\gamma}_{R \rightarrow x_{1}}=\frac{\left|\hat{h}_{1}\right|^{2} a_{1}}{\kappa_{r}^{2}\left|\hat{h}_{1}\right|^{2}+\left|\hat{h}_{2}\right|^{2}\left(a_{2}+\kappa_{r}^{2}\right)+\left|\hat{h}_{3}\right|^{2}\left(\varpi_{1} a_{3}+\kappa_{r}^{2}\right)+\left|\hat{h}_{4}\right|^{2}\left(\varpi_{1} a_{4}+\kappa_{r}^{2}\right)+\bar{\vartheta}_{1}} \\
\bar{\gamma}_{R \rightarrow x_{2}}=\frac{\left|\hat{h}_{2}\right|^{2} a_{2}}{\kappa_{r}^{2}\left|\hat{h}_{1}\right|^{2}+\kappa_{r}^{2}\left|\hat{h}_{2}\right|^{2}+\left|\hat{h}_{3}\right|^{2}\left(\varpi_{1} a_{3}+\kappa_{r}^{2}\right)+\left|\hat{h}_{4}\right|^{2}\left(\varpi_{1} a_{4}+\kappa_{r}^{2}\right)+\bar{\vartheta}_{1}}
\end{gathered}
$$

where $\bar{\vartheta}_{1}=a_{1} \delta_{e_{1}}^{2}+a_{2} \delta_{e_{2}}^{2}+\varpi_{1} a_{3} \delta_{e_{3}}^{2}+\varpi_{1} a_{4} \delta_{e_{4}}^{2}+k_{r}^{2}\left(\delta_{e_{1}}^{2}+\delta_{e_{2}}^{2}+\delta_{e_{3}}^{2}+\delta_{e_{4}}^{2}\right)$.

The asymptotic SINR of (7), (8) and (9) can be approximately expressed as

$$
\begin{gathered}
\bar{\gamma}_{U_{3} \rightarrow x_{2}}=\frac{\left|\hat{h}_{3}\right|^{2} b_{2}}{\left|\hat{h}_{3}\right|^{2} b_{1}+\left|\hat{h}_{3}\right|^{2} \kappa_{t}^{2}+\varpi_{2}\left|\hat{h}_{3}\right|^{2}+\bar{\vartheta}_{2}} \\
\bar{\gamma}_{U_{3} \rightarrow x_{1}}=\frac{\left|\hat{h}_{3}\right|^{2} b_{1}}{\left|\hat{h}_{3}\right|^{2} \kappa_{t}^{2}+\varpi_{2}\left|\hat{h}_{3}\right|^{2}+\bar{\vartheta}_{2}} \\
\bar{\gamma}_{U_{4} \rightarrow x_{2}}=\frac{\left|\hat{h}_{4}\right|^{2} b_{2}}{\left|\hat{h}_{4}\right|^{2} b_{1}+\left|\hat{h}_{4}\right|^{2} \kappa_{t}^{2}+\varpi_{2}\left|\hat{h}_{4}\right|^{2}+\bar{\vartheta}_{3}}
\end{gathered}
$$

where $\bar{\vartheta}_{2}=\delta_{e_{3}}^{2}+\delta_{e_{3}}^{2} \kappa_{t}^{2}+\varpi_{2} \delta_{e_{3}}^{2}$ and $\bar{\vartheta}_{3}=\delta_{e_{4}}^{2}+\delta_{e_{4}}^{2} \kappa_{t}^{2}+\varpi_{2} \delta_{e_{4}}^{2}$.

Lemma 1. For the non-ideal cases $\left(\delta_{e_{i}}^{2} \neq 0, \kappa_{l} \neq 0\right)$, the asymptotic outage probability of $U_{1}$ and $U_{2}$ can be derived as following

$$
\mathrm{P}_{U_{1}}^{n i, \infty} \approx 1-e^{-\frac{\bar{\tau}_{0}}{\bar{\delta}_{3}^{2}}-\frac{\bar{\theta}_{1} \bar{\theta}_{1}}{\hat{\delta}_{1}^{2}}} \prod_{i=1}^{3} \bar{\lambda}_{i}\left(\frac{\bar{\Phi}_{1} \hat{\delta}_{1}^{2}}{\bar{\lambda}_{1} \hat{\delta}_{1}^{2}+\bar{\theta}_{1}}-\frac{\bar{\Phi}_{2} \hat{\delta}_{1}^{2}}{\bar{\lambda}_{2} \hat{\delta}_{1}^{2}+\bar{\theta}_{1}}+\frac{\bar{\Phi}_{3} \hat{\delta}_{1}^{2}}{\bar{\lambda}_{3} \hat{\delta}_{1}^{2}+\bar{\theta}_{1}}\right)
$$

where $\bar{\Phi}_{1}=\left(\bar{\lambda}_{2}-\bar{\lambda}_{1}\right)^{-1}\left(\bar{\lambda}_{3}-\bar{\lambda}_{1}\right)^{-1}, \bar{\Phi}_{2}=\left(\bar{\lambda}_{3}-\bar{\lambda}_{2}\right)^{-1}\left(\bar{\lambda}_{2}-\bar{\lambda}_{1}\right)^{-1}, \bar{\Phi}_{3}=\left(\bar{\lambda}_{3}-\bar{\lambda}_{1}\right)^{-1}\left(\bar{\lambda}_{3}-\bar{\lambda}_{2}\right)^{-1}$. $\bar{\lambda}_{1}=1 /\left(\hat{\delta}_{2}^{2}\left(a_{2}+\kappa_{r}^{2}\right)\right), \bar{\lambda}_{2}=1 /\left(\hat{\delta}_{3}^{2}\left(\varpi_{1} a_{3}+\kappa_{r}^{2}\right)\right), \bar{\lambda}_{3}=1 /\left(\hat{\delta}_{4}^{2}\left(\varpi_{1} a_{4}+\kappa_{r}^{2}\right)\right) \cdot \bar{\theta}_{1}=\frac{\gamma_{t h f}}{\left(a_{1}-\gamma_{t h f} \kappa_{r}^{2}\right)}$ with $a_{1}>\gamma_{\text {thf }} \kappa_{r}^{2}, \bar{\tau}_{0}=\max \left(\bar{\tau}_{2}, \bar{\tau}_{1}\right), \bar{\tau}_{1}=\frac{\gamma_{\text {thf }} \bar{\vartheta}_{2}}{\left(b_{1}-\gamma_{\text {thf }}\left(\kappa_{t}^{2}+\varpi_{2}\right)\right)}$ with $b_{1}>\gamma_{\text {thf }}\left(\kappa_{t}^{2}+\varpi_{2}\right)$ and $\bar{\tau}_{2}=\frac{\gamma_{t h m} \bar{\vartheta}_{2}}{\left(b_{2}-\gamma_{t h m}\left(b_{1}+\kappa_{t}^{2}+\varpi_{2}\right)\right)}$ with $b_{2}>\gamma_{t h m}\left(b_{1}+\kappa_{t}^{2}+\varpi_{2}\right)$.

$$
\begin{aligned}
& \mathrm{P}_{U_{2}}^{n i, \infty} \approx 1-e^{-\left(\frac{\bar{\theta}_{2} \bar{\vartheta}_{1}}{\hat{\delta}_{2}^{2}+}+\frac{\bar{\theta}_{1} \bar{q}_{1}}{\hat{\delta}_{1}^{2}}+\frac{\bar{c}_{2}}{\hat{\delta}_{3}^{2}}+\frac{\bar{z}_{3}}{\hat{\delta}_{4}^{2}}\right)} \frac{\hat{\delta}_{2}^{2} \hat{\delta}_{1}^{2} \bar{\lambda}_{1}^{\prime} \bar{\lambda}_{2}^{\prime}}{\left(\theta_{2} \kappa_{r}^{2} \hat{\delta}_{1}^{2}+\hat{\delta}_{2}^{2}\right)\left(\theta_{1} \hat{\delta}_{2}^{2}\left(a_{2}+\kappa_{r}^{2}\right)+\hat{\delta}_{1}^{2}\right)\left(\bar{\lambda}_{2}^{\prime}-\bar{\lambda}_{1}^{\prime}\right)} \\
& \times\left(\frac{\hat{\delta}_{1}^{2} \hat{\delta}_{2}^{2}}{\bar{\theta}_{2} \hat{\delta}_{1}^{2}+\bar{\theta}_{1} \hat{\delta}_{2}^{2}+\bar{\lambda}_{1} \hat{\delta}_{1}^{2} \hat{\delta}_{2}^{2}}-\frac{\hat{\delta}_{1}^{2} \hat{\delta}_{2}^{2}}{\bar{\theta}_{2} \hat{\delta}_{1}^{2}+\bar{\theta}_{1} \hat{\delta}_{2}^{2}+\bar{\lambda}_{2}^{\prime} \hat{\delta}_{1}^{2} \hat{\delta}_{2}^{2}}\right)
\end{aligned}
$$


where $\bar{\lambda}_{1}^{\prime}=1 /\left(\hat{\delta}_{3}^{2}\left(\varpi_{1} a_{3}+\kappa_{r}^{2}\right)\right), \bar{\lambda}_{2}^{\prime}=1 /\left(\hat{\delta}_{4}^{2}\left(\varpi_{1} a_{4}+\kappa_{r}^{2}\right)\right) . \bar{\theta}_{2}=\frac{\gamma_{t h m}}{a_{2}-\gamma_{t h m} \kappa_{r}^{2}}$ with $a_{2}>\gamma_{t h m} \kappa_{r}^{2}$. $\bar{\tau}_{3}=\frac{\gamma_{t h m} \bar{\vartheta}_{3}}{b_{2}-\gamma_{t h m}\left(b_{1}+\kappa_{t}^{2}+\varpi_{2}\right)}$ with $b_{2}>\gamma_{t h m}\left(b_{1}+\kappa_{t}^{2}+\varpi_{2}\right)$.

Lemma 2. Based on the derived results of (12) and (15), when $\rho_{u}=\varepsilon \rho_{r} \rightarrow \infty$, and $\varepsilon>0$, using $e^{-x}=1-x$ and L'Hospital's rule, the ideal cases $\left(\delta_{e_{i}}^{2}=0, \kappa_{l}=0\right)$ of the outage probability of $U_{1}$ and $U_{2}$ can be approximated as follows

$$
\mathrm{P}_{U_{1}}^{\mathrm{d} d, \infty} \approx 1-\prod_{i=4}^{6} \bar{\lambda}_{i}\left(\frac{\bar{\Psi}_{1} a_{1} \delta_{1}^{2}}{a_{1} \delta_{1}^{2} \bar{\lambda}_{4}+\gamma_{\text {thf }}}-\frac{\bar{\Psi}_{2} a_{1} \delta_{1}^{2}}{a_{1} \delta_{1}^{2} \bar{\lambda}_{5}+\gamma_{\text {thf }}}+\frac{\bar{\Psi}_{3} a_{1} \delta_{1}^{2}}{a_{1} \delta_{1}^{2} \bar{\lambda}_{6}+\gamma_{\text {thf }}}\right)
$$

where $\bar{\Psi}_{1}=\left(\bar{\lambda}_{5}-\bar{\lambda}_{4}\right)^{-1}\left(\bar{\lambda}_{6}-\bar{\lambda}_{4}\right)^{-1}, \bar{\Psi}_{2}=\left(\bar{\lambda}_{6}-\bar{\lambda}_{5}\right)^{-1}\left(\bar{\lambda}_{5}-\bar{\lambda}_{4}\right)^{-1}, \bar{\Psi}_{3}=\left(\bar{\lambda}_{6}-\bar{\lambda}_{4}\right)^{-1}\left(\bar{\lambda}_{6}-\bar{\lambda}_{5}\right)^{-1}$. $\bar{\lambda}_{4}=1 /\left(a_{2} \delta_{2}^{2}\right), \bar{\lambda}_{5}=1 /\left(\varpi_{1} a_{3} \delta_{3}^{2}\right)$ and $\bar{\lambda}_{6}=1 /\left(\varpi_{1} a_{4} \delta_{4}^{2}\right)$.

$$
\mathrm{P}_{U_{2}}^{i d, \infty} \approx 1-\frac{\bar{\lambda}_{5} \bar{\lambda}_{6}}{\beta_{t} \delta_{2}^{2}\left(\bar{\lambda}_{6}-\bar{\lambda}_{5}\right)} \sum_{k=5}^{6} \frac{(-1)^{k+1} a_{2}}{\beta_{t} \gamma_{t h m}+\bar{\lambda}_{k} a_{2}}
$$

where $\beta_{t}=\frac{\gamma_{\text {thf }} a_{2}}{a_{1} \delta_{1}^{2}}+\frac{1}{\delta_{2}^{2}}$.

3) Diversity Orders

In this subsection, the diversity order is analyzed, which is defined as [34]

$$
d=-\lim _{\rho \rightarrow \infty} \frac{\log \left(\mathrm{P}_{U_{n}}^{\infty}(\rho)\right)}{\log \rho}
$$

where $\mathrm{P}_{U_{n}}^{\infty}$ denotes the asymptotic outage probability of $U_{n}, n \in\{1,2\}$.

By using the definition in (25), the diversity orders for the non-ideal conditions $\left(\delta_{e_{i}}^{2} \neq 0, \kappa_{l} \neq 0\right)$ of both $U_{1}$ and $U_{2}$ are obtained as

$$
\begin{aligned}
& d_{1}^{n i}=-\lim _{\rho \rightarrow \infty} \frac{\log \left(\mathrm{P}_{U_{1}}^{n i, \infty}(\rho)\right)}{\log \rho}=0 \\
& d_{2}^{n i}=-\lim _{\rho \rightarrow \infty} \frac{\log \left(\mathrm{P}_{U_{2}}^{n i, \infty}(\rho)\right)}{\log \rho}=0
\end{aligned}
$$

The diversity orders for the ideal conditions $\left(\delta_{e_{i}}^{2}=0, \kappa_{l}=0\right)$ of both $U_{1}$ and $U_{2}$ are derived as

$$
\begin{aligned}
& d_{1}^{i d}=-\lim _{\rho \rightarrow \infty} \frac{\log \left(\mathrm{P}_{U_{1}}^{\text {id, }}(\rho)\right)}{\log \rho}=0 \\
& d_{2}^{i d}=-\lim _{\rho \rightarrow \infty} \frac{\log \left(\mathrm{P}_{U_{2}}^{\text {id, }}(\rho)\right)}{\log \rho}=0
\end{aligned}
$$


Remark: As can be observed from (21)-(24), there exists error floors for both the ideal and non-ideal conditions due to the intra-group interference. In addition, RHIs and CEEs deteriorate the outage probability since they can be recognized extra interference. As can also be seen in (26)-(29), the diversity orders for the ideal and non-ideal conditions are both zero due to the fixed outage probabilities at high SNRs. This means that TWR-NOMA can not obtain diversity gains.

\section{Numerical Results and Discussions}

In this section, some numerical results are provided to verify the accuracy of the theoretical analysis. We provide numerical illustration of our analytical results through Monte Carlo Simulations. Unless otherwise specified, the parameter values are provided in the Table 1. For convenience, we assume $\delta_{e}^{2}=\delta_{e_{1}}^{2}=\delta_{e_{2}}^{2}=\delta_{e_{3}}^{2}=\delta_{e_{4}}^{2}, \kappa=\kappa_{r}=\kappa_{t}$.

Table 1. Table of simulation parameters for numerical results

\begin{tabular}{|l|l|}
\hline Simulation parameters & Values \\
\hline Monte Carlo simulations repeated & $10^{5}$ iterations \\
\hline Power allocation coefficients of NOMA in & $a_{1}=a_{3}=0.75$, \\
the first phase & $a_{2}=a_{4}=0.25$ \\
\hline $\begin{array}{l}\text { Power allocation coefficients of NOMA in } \\
\text { the second phase }\end{array}$ & $\begin{array}{l}b_{1}=b_{3}=0.25, \\
b_{2}=b_{4}=0.75\end{array}$ \\
\hline Average gain of estimated channel for $\hat{h}_{1}, \hat{h}_{2}$ & $\hat{\delta}_{1}^{2}=d_{1}^{-\alpha}, \hat{\delta}_{2}^{2}=d_{2}^{-\alpha}$ \\
\hline The distance between R and $U_{1}$ or $U_{3}$ & $d_{1}=2 m$ \\
\hline The distance between $\mathrm{R}$ and $U_{2}$ or $U_{4}$ & $d_{2}=4 m$ \\
\hline Pass loss exponent & $\alpha=4$ \\
\hline Targeted data rates & $R_{f}=0.1 \mathrm{BPCU}$ and $R_{m}=0.01 \mathrm{BPCU}$ \\
\hline
\end{tabular}

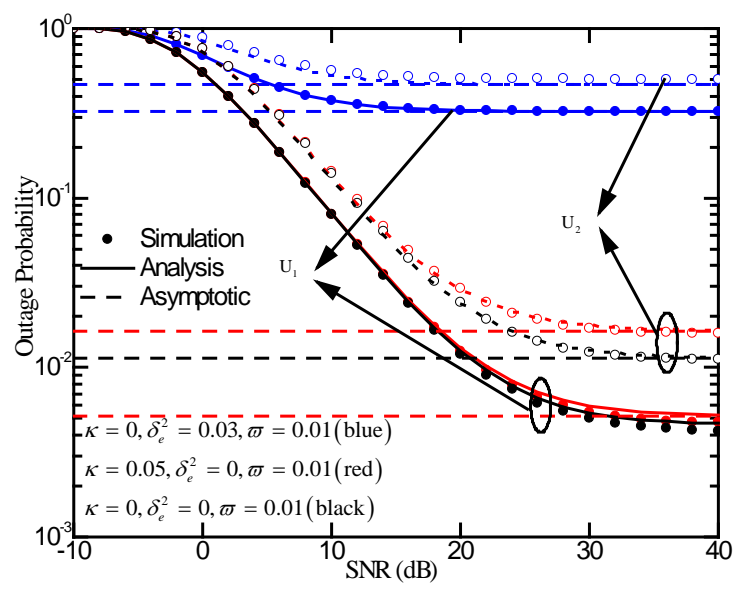

Fig. 2. Outage probability versus the transmit SNR. 
Fig. 2 plots the outage probabilities of the two users versus transmit SNR for different values of $\kappa$ and $\delta_{e}^{2}$. We consider three cases in this simulation. 1) $\kappa=0, \delta_{e}^{2}=0.03$; 2) $\kappa=0.05, \delta_{e}^{2}=0$; 3) $\kappa=0, \delta_{e}^{2}=0$. The curves represent the exact and asymptotic analytical of outage probability for $U_{1}$ and $U_{2}$ of ideal and non-ideal conditions in (11), (12), (14), (15) and (21), (22), (23), (24), respectively. It is clear that analytical curves are in good agreement with Monte Carlo simulations. It can be seen from this figure that the outage performance of the system with RHIs or CEEs is worse than that of the system without RHIs and CEEs for both $U_{1}$ and $U_{2}$, which means that RHIs and CEEs have deleterious effects on the system outage performance. Moreover, it is readily noticed from Fig. 2 that for the third case, there are error floors for $U_{1}$ and $U_{2}$. The reason can be explained that the intra-group IS result in zero diversity orders. This conclusion is confirmed by (28) and (29).

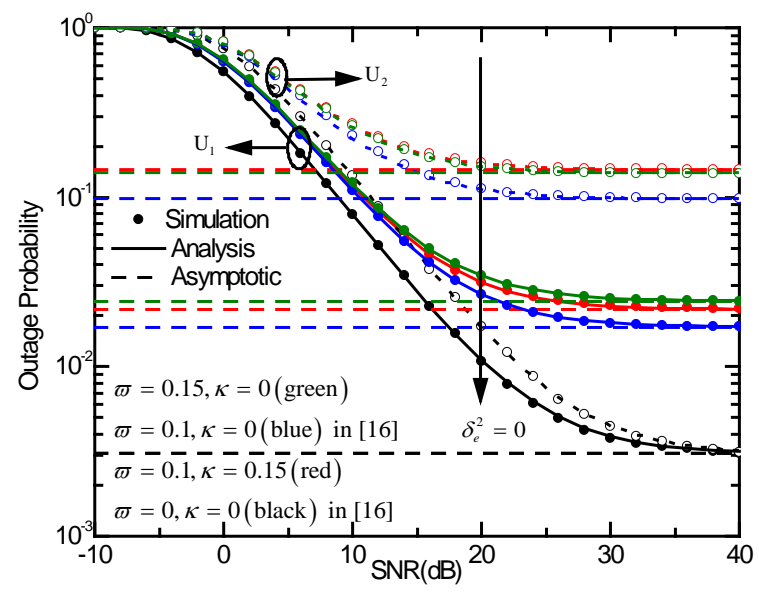

Fig. 3. Outage probability versus the transmit SNR.

Fig. 3 plots the outage performance versus SNR with different levels of inter-group IS from $\varpi=\{0,0.1,0.15\}$. For the purpose of comparison, the results of [16] have been provided. It can be seen that with the improvement of inter-group IS coefficient, the outage performance decreases obviously. We can conclude that the existence of the inter-group IS makes the outage performance worse. In addition, for the case $(\varpi=0)$ and $(\varpi=0.1)$, it still have the error floors caused by the intra-group IS, which is consistent with the results of [16]. We can observe that RHIs has a negative effect on the outage performance by comparing the red curves with the blue curves. 


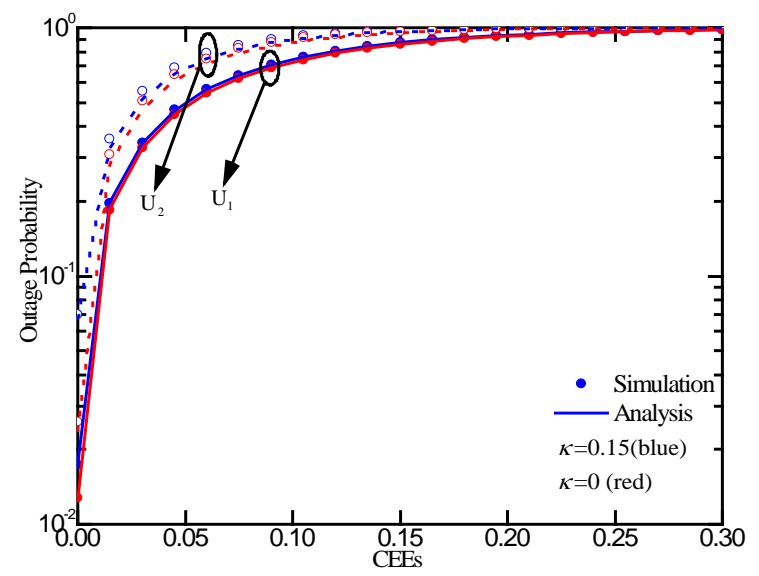

Fig. 4. Outage probability versus the CEEs.

Fig. 4 plots the outage performance of the TWR-NOMA system versus CEEs for different RHIs parameters $\kappa=\{0,0.15\}$. As observed from Fig. 4, the outage probabilities of the TWR-NOMA network for the two users increase as CEEs grow large. This happens because the CEEs act as interference for the desired signal, which reduces the system performance. Moreover, the growth of $U_{1}$ is larger than that of $U_{2}$, which means that CEEs have more serious effect on the outage probabilities of the near users than the far users for this system.

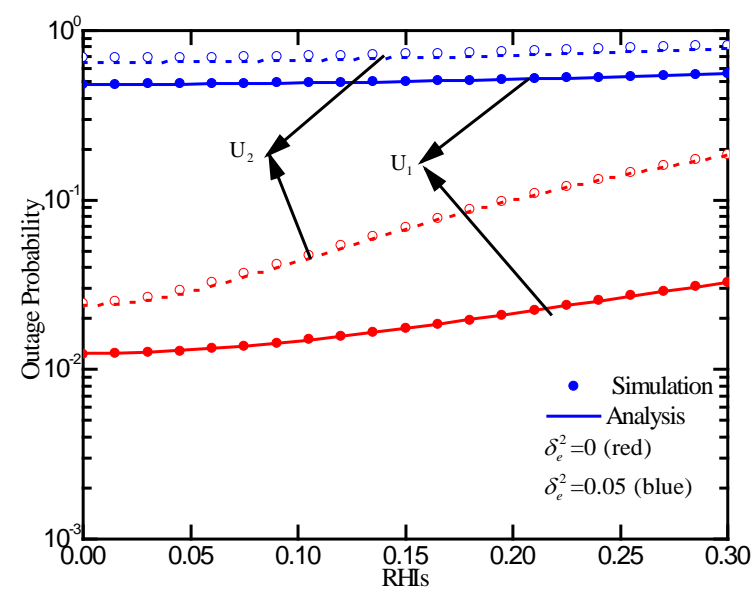

Fig. 5. Outage probability versus the RHIs at the relay node.

Fig. 5 illustrates the impact of the RHIs at the relay node on the outage performance of the TWR-NOMA systems. These curves represent two cases: ideal case $\left(\delta_{e}^{2}=0\right)$ and non-ideal case $\left(\delta_{e}^{2}=0.05\right)$. In addition, we assume a fixed transmit SNR (20dB) in this simulation. It can be observed that the outage performance becomes worse as the RHIs increase in both two 
cases. Moreover, when the RHIs is equal to zero $(\kappa=0)$, the outage probabilities of this two cases have different values, due to the CEEs existence in the non-ideal case.

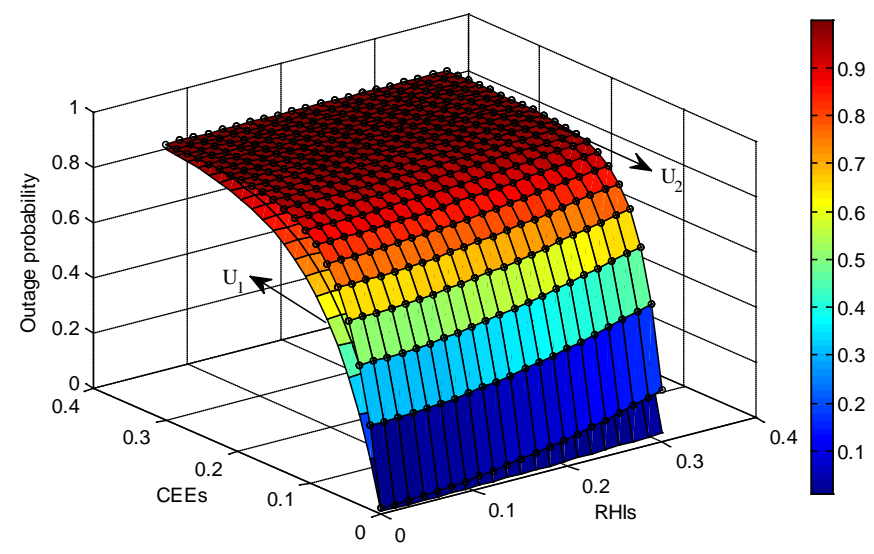

Fig. 6. Outage probability versus the RHIs and CEEs.

Fig. 6 illustrates the impact of the RHIs and the CEEs at the relay node on the outage performance of the TWR-NOMA systems. We use the change of color to reflect the outage performance affected. There is a chromaticity bar on the right side of the graph. As the color changes from dark to light indicates that the performance of system outage becomes degrades. There are two layers of grid in this figure, the lower one denotes the performance of $U_{1}$ and the other layer denotes the performance of $U_{2}$. This means that the performance of $U_{1}$ is better than that of $U_{2}$. It is apparent to see from the Fig. $\mathbf{6}$ that as RHIs increase, the color changes from dark blue to light blue, and as the CEEs increase, the color clearly changes from dark to light, which means the outage probability becomes worse, respectively.

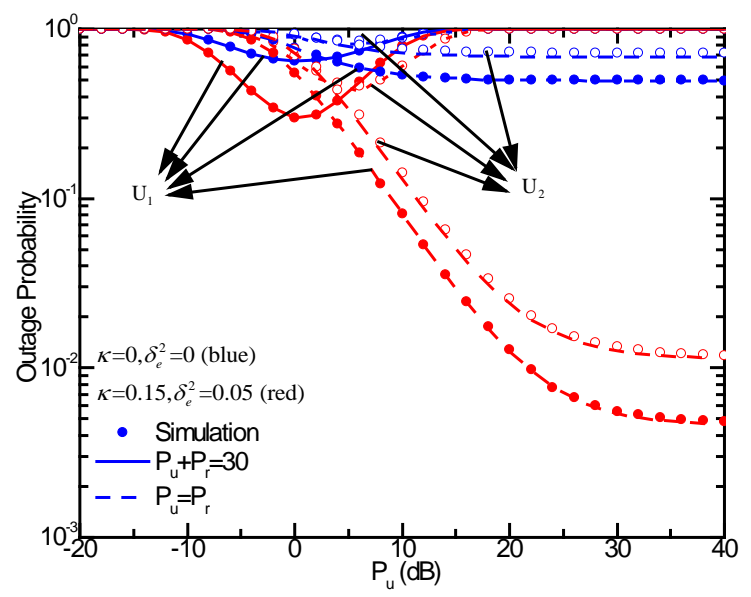

Fig. 7. Outage probability versus $P_{u}$ 
Fig. 7 depicts the impact of $P_{u}$ on the outage performance. In this simulation, we consider two cases: 1) $P_{u}=-20: 40 \mathrm{~dB}$ and $P_{u}+P_{r}=30 \mathrm{~dB}$; 2) $P_{u}=-20: 40 \mathrm{~dB}$ and $P_{u}=P_{r}$. For the first case, we can clearly see that there exist optimal allocation powers (about $0 \mathrm{~dB}$ for $U_{1}$ and $6 \mathrm{~dB}$ for $U_{2}$ ) for $P_{u}$ of $U_{1}$ and $U_{2}$. This happens because the performance gain caused by increasing the power of users $\left(P_{u}\right)$ is larger than the performance loss caused by reducing the power of relay $\left(P_{r}\right)$. For the second case, we can also see that the outage probabilities for the two users always decrease as the power of users and relay increasing.

\section{Conclusion}

This paper investigated the performance of TWR-NOMA networks with RHIs and CEEs, in which two groups of users exchange information with the aid of a DF relay. The closed-form expressions for exact outage probability of two group users were derived and the asymptotic behavior was discussed. Based on the derived analytical results, we further evaluated the diversity orders achieved by the users. Results revealed that CEEs have more deleterious effects on the outage probabilities of the near users than the far users. Furthermore, it was shown that the TWR-NOMA networks with inter-group IS, intra-group IS, RHIs or CEEs cause error floors.

\section{APPENDIX A}

\section{PROOF OF THEOREM 1}

It is worth noting that for the convenience of calculation, we assume $X_{i}=\left|h_{i}\right|^{2}, \quad \hat{X}_{i}=\left|\hat{h}_{i}\right|^{2}$ with $i \in\{1,2,3,4\}$. Substituting (3), (7) and (8) into (10), the outage probability of $U_{1}$ is given by

$$
\begin{aligned}
& \mathrm{P}_{U_{1}}^{n i, \text { out }}=1-\operatorname{Pr}\left(\gamma_{R \rightarrow x_{1}}>\gamma_{\text {thf }}\right) \operatorname{Pr}\left(\gamma_{U_{3} \rightarrow x_{2}}>\gamma_{\text {thm }}, \gamma_{U_{3} \rightarrow x_{1}}>\gamma_{\text {thf }}\right) \\
& =1-f_{1} f_{2} \\
& f_{1}=\operatorname{Pr}\left(\frac{\rho_{u}\left|\hat{h}_{1}\right|^{2} a_{1}}{\rho_{u}\left|\hat{h}_{2}\right|^{2} a_{2}+\sum_{k=1}^{4} \rho_{u}\left|\hat{h}_{k}\right|^{2} \kappa_{r}^{2}+\sum_{j=3}^{4} \rho_{u}\left|\hat{h}_{j}\right|^{2} \varpi_{1} a_{j}+\vartheta_{1}}>\gamma_{\text {thf }}\right), \\
& f_{2}=\operatorname{Pr}\left(\frac{\rho_{r}\left|\hat{h}_{3}\right|^{2} b_{2}}{\rho_{r}\left|\hat{h}_{3}\right|^{2} b_{1}+\rho_{r}\left|\hat{h}_{3}\right|^{2} \kappa_{t}^{2}+\varpi_{2} \rho_{r}\left|\hat{h}_{3}\right|^{2}+\vartheta_{2}}>\gamma_{t h m}\right. \text {, } \\
& \left.\frac{\rho_{r}\left|\hat{h}_{3}\right|^{2} b_{1}}{\rho_{r}\left|\hat{h}_{3}\right|^{2} \kappa_{t}^{2}+\varpi_{2} \rho_{r}\left|\hat{h}_{3}\right|^{2}+\vartheta_{2}}>\gamma_{\text {thf }}\right),
\end{aligned}
$$


For calculating the probability of $f_{1}$ in (A.2), we set $z=z_{1}+z_{2}+z_{3}$ with $z_{1}=\rho_{u}\left|\hat{h}_{2}\right|^{2}\left(a_{2}+\kappa_{r}^{2}\right), z_{2}=\rho_{u}\left|\hat{h}_{3}\right|^{2}\left(\varpi_{1} a_{3}+\kappa_{r}^{2}\right)$, and $z_{3}=\rho_{u}\left|\hat{h}_{4}\right|^{2}\left(\varpi_{1} a_{4}+\kappa_{r}^{2}\right)$. As we wrote earlier, $\left|\hat{h}_{i}\right|^{2}$ follow the exponential distribution with the means $\hat{\delta}_{i}^{2}, i \in\{1,2,3,4\}$. In addition, $z_{1}, z_{2}$, and $z_{3}$ are also independent exponentially distributed random variables with means $\lambda_{1}=1 /\left(\rho_{u} \hat{\delta}_{2}^{2}\left(a_{2}+\kappa_{r}^{2}\right)\right), \lambda_{2}=1 /\left(\rho_{u} \hat{\delta}_{3}^{2}\left(\varpi_{1} a_{3}+\kappa_{r}^{2}\right)\right)$, and $\lambda_{3}=1 /\left(\rho_{u} \hat{\delta}_{4}^{2}\left(\varpi_{1} a_{4}+\kappa_{r}^{2}\right)\right)$, respectively. As shown in the [35], for the independent non-identical distributed fading scenario, the PDF of $z$ is given by

$$
f_{Z}(z)=\prod_{t=1}^{3} \lambda_{t}\left(\Phi_{1} e^{-\lambda_{1} z}-\Phi_{2} e^{-\lambda_{2} z}+\Phi_{3} e^{-\lambda_{3} z}\right),
$$

where $\Phi_{1}=\left(\lambda_{2}-\lambda_{1}\right)^{-1}\left(\lambda_{3}-\lambda_{1}\right)^{-1}, \Phi_{2}=\left(\lambda_{3}-\lambda_{2}\right)^{-1}\left(\lambda_{2}-\lambda_{1}\right)^{-1}, \Phi_{3}=\left(\lambda_{3}-\lambda_{1}\right)^{-1}\left(\lambda_{3}-\lambda_{2}\right)^{-1}$.

According to the above explanations, $f_{1}$ is expressed as follows

$$
\begin{aligned}
f_{1} & =\operatorname{Pr}\left(\hat{X}_{1}>\theta_{1}\left(z+\vartheta_{1}\right)\right) \\
& =e^{-\frac{\theta_{1} \vartheta_{1}}{\hat{\delta}_{1}^{2}}} \prod_{i=1}^{3} \lambda_{i}\left(\frac{\Phi_{1} \hat{\delta}_{1}^{2}}{\lambda_{1} \hat{\delta}_{1}^{2}+\theta_{1}}-\frac{\Phi_{2} \hat{\delta}_{1}^{2}}{\lambda_{2} \hat{\delta}_{1}^{2}+\theta_{1}}+\frac{\Phi_{3} \hat{\delta}_{1}^{2}}{\lambda_{3} \hat{\delta}_{1}^{2}+\theta_{1}}\right),
\end{aligned}
$$

where $\theta_{1}=\frac{\gamma_{\text {thf }}}{\rho_{u}\left(a_{1}-\gamma_{\text {thf }} \kappa_{r}^{2}\right)}$, with $a_{1}>\gamma_{\text {thf }} \kappa_{r}^{2}$.

$f_{2}$ can be further calculated as follows

$$
\begin{aligned}
f_{2} & =\operatorname{Pr}\left(\hat{X}_{3}>\tau_{2}, \hat{X}_{3}>\tau_{1}\right) \\
& =\operatorname{Pr}\left(\hat{X}_{3}>\max \left(\tau_{2}, \tau_{1}\right)=\tau_{0}\right) \\
& =e^{-\frac{\tau_{0}}{\hat{\delta}_{3}^{2}}},
\end{aligned}
$$

where $\tau_{0}=\max \left(\tau_{2}, \tau_{1}\right), \tau_{2}=\frac{\gamma_{\text {thm }} \vartheta_{2}}{\rho_{r}\left(b_{2}-\gamma_{\text {thm }}\left(b_{1}+\kappa_{t}^{2}+\varpi_{2}\right)\right)}$ with $b_{2}>\gamma_{\text {thm }}\left(b_{1}+\kappa_{t}^{2}+\varpi_{2}\right)$ and $\tau_{1}=\frac{\gamma_{t h f} \vartheta_{2}}{\rho_{r}\left(b_{1}-\gamma_{t h f}\left(\kappa_{t}^{2}+\varpi_{2}\right)\right)}$ with $b_{1}>\gamma_{t h f}\left(\kappa_{t}^{2}+\varpi_{2}\right)$.

Substituting (A.5), (A.6) into (A.1), (11) can be obtained.

The proof is completed.

\section{APPENDIX B}

\section{PROOF OF COROLLARY 1}

The outage probability of $U_{1}$ for the ideal cases can be expressed as 


$$
\begin{aligned}
\mathrm{P}_{U_{1}}^{\text {id,out }} & =1-\operatorname{Pr}\left(\gamma_{R \rightarrow x_{1}}>\gamma_{\text {thf }}\right) \operatorname{Pr}\left(\gamma_{U_{3} \rightarrow x_{2}}>\gamma_{t h m}, \gamma_{U_{3} \rightarrow x_{1}}>\gamma_{\text {thf }}\right) \\
& =1-\operatorname{Pr}\left(\frac{\rho_{u} X_{1} a_{1}}{\rho_{u} X_{2} a_{2}+\varpi_{1} \rho_{u} X_{3} a_{3}+\varpi_{1} \rho_{u} X_{4} a_{4}+1}>\gamma_{t h f}\right) \\
& \times \operatorname{Pr}\left(\frac{\rho_{r} X_{3} b_{2}}{\rho_{r} X_{3}\left(b_{1}+\varpi_{2}\right)+1}>\gamma_{t h m}, \frac{\rho_{r} X_{3} b_{1}}{\rho_{r} X_{3} \varpi_{2}+1}>\gamma_{t h f}\right) \\
& =1-\operatorname{Pr}\left(X_{1}>\frac{\gamma_{t h f}}{\rho_{u} a_{1}}(Y+1)\right) \operatorname{Pr}\left(X_{3}>\tau_{0}^{\prime}=\max \left(\tau_{2}^{\prime}, \tau_{1}^{\prime}\right)\right) \\
& =1-f_{1}^{\prime} f_{2}^{\prime},
\end{aligned}
$$

where $Y=Y_{4}+Y_{5}+Y$, with $Y_{4}=\rho_{u} a_{2} X_{2}, Y_{5}=\varpi_{1} \rho_{u} a_{3} X_{3}, Y_{6}=\varpi_{1} \rho_{u} a_{4} X_{4}$. As we wrote earlier $Y_{4}, Y_{5}$ and $Y_{6}$ are independent exponentially distributed random variables with means $\lambda_{4}=1 /\left(\rho_{u} a_{2} \delta_{2}^{2}\right), \lambda_{5}=1 /\left(\varpi_{1} \rho_{u} a_{3} \delta_{3}^{2}\right)$, and $\lambda_{6}=1 /\left(\varpi_{1} \rho_{u} a_{4} \delta_{4}^{2}\right)$.

$f_{1}^{\prime}$ can be further expressed as follows

$$
\begin{aligned}
& f_{1}^{\prime}=\operatorname{Pr}\left(X_{1}>\frac{\gamma_{\text {thf }}}{\rho_{u} a_{1}}(Y+1)\right) \\
& =e^{-\frac{\gamma_{\text {thf }}}{\xi_{1}}} \prod_{i=1}^{3} \lambda_{i}\left(\frac{\Psi_{1} \xi_{1}}{\xi_{1} \lambda_{4}+\gamma_{\text {thf }}}-\frac{\Psi_{2} \xi_{1}}{\xi_{1} \lambda_{5}+\gamma_{\text {thf }}}+\frac{\Psi_{3} \xi_{1}}{\xi_{1} \lambda_{6}+\gamma_{\text {thf }}}\right),
\end{aligned}
$$

where $\Psi_{1}=\left(\lambda_{5}-\lambda_{4}\right)^{-1}\left(\lambda_{6}-\lambda_{4}\right)^{-1}, \Psi_{2}=\left(\lambda_{6}-\lambda_{5}\right)^{-1}\left(\lambda_{5}-\lambda_{4}\right)^{-1}, \Psi_{3}=\left(\lambda_{6}-\lambda_{4}\right)^{-1}\left(\lambda_{6}-\lambda_{5}\right)^{-1}$. $\xi_{1}=\rho_{u} a_{1} \delta_{1}^{2}$.

$f_{2}^{\prime}$ can be easily calculated as follows

$$
f_{2}^{\prime}=\operatorname{Pr}\left(X_{3}>\tau_{0}^{\prime}\right)=e^{-\frac{\tau_{0}^{\prime}}{\delta_{3}^{2}}}
$$

where $\tau_{0}^{\prime}=\max \left(\tau_{2}^{\prime}, \tau_{1}^{\prime}\right), \tau_{1}^{\prime}=\frac{\gamma_{t h f}}{\rho_{r}\left(b_{1}-\gamma_{t h f} \varpi_{2}\right)}$ with $b_{1}>\gamma_{t h f} \varpi_{2}, \tau_{2}^{\prime}=\frac{\gamma_{t h m}}{\rho_{r}\left(b_{2}-\gamma_{t h m}\left(b_{1}+\varpi_{2}\right)\right)}$ with $b_{2}>\gamma_{\text {thm }}\left(b_{1}+\varpi_{2}\right)$.

Combining (B.2), (B.3) into (B.1), we can obtain (12).

The proof is completed.

\section{APPENDIX C}

\section{PROOF OF THEOREM 2}

The outage probability of $U_{2}$ can be expressed as

$$
\begin{aligned}
\mathrm{P}_{U_{2}}^{\text {ni,out }} & =1-\operatorname{Pr}\left(\gamma_{R \rightarrow x_{2}}>\gamma_{\text {thm }}, \gamma_{R \rightarrow x_{1}}>\gamma_{\text {thf }}\right) \operatorname{Pr}\left(\gamma_{u_{3} \rightarrow x_{2}}>\gamma_{\text {thm }}\right) \operatorname{Pr}\left(\gamma_{u_{4} \rightarrow x_{2}}>\gamma_{\text {thm }}\right) \\
& =1-f_{3} f_{4} f_{5}
\end{aligned}
$$


Substituting (3), (4) into (C.1), $f_{3}$ can be further given by

$$
\begin{aligned}
f_{3} & =\operatorname{Pr}\left(\gamma_{R \rightarrow x_{2}}>\gamma_{t h m}, \gamma_{R \rightarrow x_{1}}>\gamma_{t h f}\right) \\
& =\operatorname{Pr}\left(\frac{\rho_{u}\left|\hat{h}_{2}\right|^{2} a_{2}}{\sum_{k=1}^{4} \rho_{u}\left|\hat{h}_{k}\right|^{2} \kappa_{r}^{2}+\sum_{j=3}^{4} \rho_{u}\left|\hat{h}_{j}\right|^{2} \varpi_{1} a_{j}+\vartheta_{1}}>\gamma_{t h m},\right. \\
& \left.\frac{\rho_{u}\left|\hat{h}_{1}\right|^{2} a_{1}}{\rho_{u}\left|\hat{h}_{2}\right|^{2} a_{2}+\sum_{k=1}^{4} \rho_{u}\left|\hat{h}_{k}\right|^{2} \kappa_{r}^{2}+\sum_{j=3}^{4} \rho_{u}\left|\hat{h}_{j}\right|^{2} \varpi_{1} a_{j}+\vartheta_{1}}>\gamma_{t h f}\right) \\
= & \operatorname{Pr}\left(\hat{X}_{2}>\theta_{2}\left(\kappa_{r}^{2} \rho_{u} \hat{X}_{1}+z^{\prime}+\vartheta_{1}\right), \hat{X}_{1}>\theta_{1}\left(\rho_{u}\left(a_{2}+\kappa_{r}^{2}\right) \hat{X}_{2}+z^{\prime}+\vartheta_{1}\right)\right) \\
= & e^{-\left(\frac{\theta_{2} \vartheta_{1}}{\hat{\delta}_{2}^{2}}+\frac{\theta_{1} \vartheta_{1}}{\hat{\delta}_{1}^{2}}\right)} \frac{\hat{\delta}_{2}^{2} \hat{\delta}_{1}^{2}}{\left(\theta_{2} k_{r}^{2} \rho_{u} \hat{\delta}_{1}^{2}+\hat{\delta}_{2}^{2}\right)\left(\theta_{1} \rho_{u} \hat{\delta}_{2}^{2}\left(a_{2}+k_{r}^{2}\right)+\hat{\delta}_{1}^{2}\right)} \int_{0}^{\infty} e^{-\left(\frac{\theta_{2}}{\hat{\delta}_{2}^{2}}+\frac{\theta_{1}}{\delta_{1}^{2}}\right) z^{\prime}} f\left(z^{\prime}\right) d z^{\prime} .
\end{aligned}
$$

For calculating the probability of $f_{3}$ in (C.1), we assume $z^{\prime}=z_{1}^{\prime}+z_{2}^{\prime}$, with $z_{1}^{\prime}=\rho_{u}\left|\hat{h}_{3}\right|^{2}\left(\varpi_{1} a_{3}+\kappa_{r}^{2}\right)$, and $z_{2}^{\prime}=\rho_{u}\left|\hat{h}_{4}\right|^{2}\left(\varpi_{1} a_{4}+\kappa_{r}^{2}\right)$. $z_{1}^{\prime}$ and $z_{2}^{\prime}$ are also independent exponentially distributed random variables with means $\lambda_{1}^{\prime}=1 /\left(\rho_{u} \hat{\delta}_{3}^{2}\left(\varpi_{1} a_{3}+\kappa_{r}^{2}\right)\right)$ and $\lambda_{2}^{\prime}=1 /\left(\rho_{u} \hat{\delta}_{4}^{2}\left(\varpi_{1} a_{4}+\kappa_{r}^{2}\right)\right)$, respectively. For the independent non-identical distributed fading scenario, the PDF of $z^{\prime}$ is given by

$$
f_{z^{\prime}}\left(z^{\prime}\right)=\prod_{t=1}^{2} \lambda_{i}^{\prime}\left(\frac{e^{-\lambda_{1} z^{\prime}}}{\lambda_{2}^{\prime}-\lambda_{1}^{\prime}}-\frac{e^{-\lambda_{2} z^{\prime}}}{\lambda_{2}^{\prime}-\lambda_{1}^{\prime}}\right) .
$$

Substituting (C.3) into (C.2), $f_{3}$ can be further rewritten as

$$
\begin{aligned}
f_{3}= & e^{-\left(\frac{\theta_{2} \vartheta_{1}}{\hat{\delta}_{2}^{2}}+\frac{\theta_{1} \vartheta_{1}}{\hat{\delta}_{1}^{2}}\right)} \frac{\hat{\delta}_{2}^{2} \hat{\delta}_{1}^{2} \lambda_{1}^{\prime} \lambda_{2}^{\prime}}{\left(\theta_{2} k_{r}^{2} \rho_{u} \hat{\delta}_{1}^{2}+\hat{\delta}_{2}^{2}\right)\left(\theta_{1} \rho_{u} \hat{\delta}_{2}^{2}\left(a_{2}+k_{r}^{2}\right)+\hat{\delta}_{1}^{2}\right)\left(\lambda_{2}^{\prime}-\lambda_{1}^{\prime}\right)} \\
& \times\left(\frac{\hat{\delta}_{1}^{2} \hat{\delta}_{2}^{2}}{\theta_{2} \hat{\delta}_{1}^{2}+\theta_{1} \hat{\delta}_{2}^{2}+\lambda_{1}^{\prime} \hat{\delta}_{1}^{2} \hat{\delta}_{2}^{2}}-\frac{\hat{\delta}_{1}^{2} \hat{\delta}_{2}^{2}}{\theta_{2} \hat{\delta}_{1}^{2}+\theta_{1} \hat{\delta}_{2}^{2}+\lambda_{2}^{\prime} \hat{\delta}_{1}^{2} \hat{\delta}_{2}^{2}}\right) .
\end{aligned}
$$

Substituting (7), (9) into (C.1), $f_{4}$ and $f_{5}$ can be calculated as follows

$$
\begin{aligned}
f_{4} & =\operatorname{Pr}\left(\frac{\rho_{r}\left|\hat{h}_{3}\right|^{2} b_{2}}{\rho_{r}\left|\hat{h}_{3}\right|^{2} b_{1}+\rho_{r}\left|\hat{h}_{3}\right|^{2} k_{t}^{2}+\varpi_{2} \rho_{r}\left|\hat{h}_{3}\right|^{2}+\vartheta_{2}}>\gamma_{t h m}\right) \\
& =\operatorname{Pr}\left(\hat{X}_{3}>\tau_{2}\right) \\
& =e^{-\frac{\tau_{2}}{\delta_{3}^{2}}} .
\end{aligned}
$$




$$
\begin{aligned}
f_{5} & =\operatorname{Pr}\left(\frac{\rho_{r}\left|\hat{h}_{4}\right|^{2} b_{2}}{\rho_{r}\left|\hat{h}_{4}\right|^{2} b_{1}+\rho_{r}\left|\hat{h}_{4}\right|^{2} \kappa_{t}^{2}+\varpi_{2} \rho_{r}\left|\hat{h}_{4}\right|^{2}+\vartheta_{3}}>\gamma_{t h m}\right) \\
& =\operatorname{Pr}\left(\hat{X}_{4}>\tau_{3}\right) \\
& =e^{-\frac{\tau_{3}}{\hat{\delta}_{4}^{2}}},
\end{aligned}
$$

where $\tau_{3}=\frac{\gamma_{t h m} \vartheta_{3}}{\rho_{r}\left(b_{2}-\gamma_{t h m}\left(b_{1}+\kappa_{t}^{2}+\varpi_{2}\right)\right)}$, with $b_{2}>\gamma_{t h m}\left(b_{1}+\kappa_{t}^{2}+\varpi_{2}\right)$. Finally, (14) can be obtained by combing (C.4), (C.5), and (C.6).

The proof is completed.

\section{APPENDIX D}

\section{PROOF OF COROLLARY 2}

The outage probability of $U_{2}$ with ideal conditions can be expressed as

$$
\begin{aligned}
\mathrm{P}_{U_{2}}^{\text {id, out }} & =1-\operatorname{Pr}\left(\gamma_{R \rightarrow x_{2}}>\gamma_{\text {thm }}, \gamma_{R \rightarrow x_{1}}>\gamma_{\text {thf }}\right) \operatorname{Pr}\left(\gamma_{U_{3} \rightarrow x_{2}}>\gamma_{\text {thm }}\right) \operatorname{Pr}\left(\gamma_{U_{4} \rightarrow x_{2}}>\gamma_{\text {thm }}\right) \\
& =1-f_{3}^{\prime} f_{4}^{\prime} f_{5}^{\prime}
\end{aligned}
$$

$f_{3}^{\prime}$ can be further calculated as

$$
\begin{aligned}
& f_{3}^{\prime}=\operatorname{Pr}\left(\frac{\rho_{u}\left|h_{2}\right|^{2} a_{2}}{\rho_{u}\left|h_{3}\right|^{2} \varpi_{1} a_{3}+\rho_{u}\left|h_{4}\right|^{2} \varpi_{1} a_{4}+1}>\gamma_{t h m}, \frac{\rho_{u}\left|h_{1}\right|^{2} a_{1}}{\rho_{u}\left|h_{2}\right|^{2} a_{2}+\rho_{u}\left|h_{3}\right|^{2} \varpi_{1} a_{3}+\rho_{u}\left|h_{4}\right|^{2} \varpi_{1} a_{4}+1}>\gamma_{\text {thf }}\right) \\
& =\operatorname{Pr}\left(X_{2}>\frac{\gamma_{\text {thm }}}{\rho_{u} a_{2}}\left(Y^{\prime}+1\right), X_{1}>\frac{\gamma_{\text {thf }}}{\rho_{u} a_{1}}\left(\rho_{u} a_{2} X_{2}+Y^{\prime}+1\right)\right) \\
& =\frac{1}{\beta_{t} \delta_{2}^{2}} e^{-\left(\frac{\gamma_{t h m} \beta_{t}}{\rho_{u} a_{2}}+\frac{\gamma_{\text {thf }}}{\rho_{u} a_{1} \delta_{1}^{2}}\right)} \int_{0}^{\infty} e^{-\frac{\gamma_{t h m} \beta_{t}}{\rho_{u} a_{2}} Y^{\prime}} f\left(Y^{\prime}\right) d Y^{\prime} \\
& =\frac{\lambda_{5} \lambda_{6}}{\beta_{t} \delta_{2}^{2}\left(\lambda_{6}-\lambda_{5}\right)} e^{-\left(\frac{\gamma_{t h m} \beta_{t}}{\rho_{u} a_{2}}+\frac{\gamma_{t h t}}{\rho_{u} a_{1} \delta_{1}^{2}}\right)} \sum_{k=5}^{6} \frac{(-1)^{k+1} \rho_{u} a_{2}}{\beta_{t} \gamma_{t h m}+\lambda_{k} \rho_{u} a_{2}},
\end{aligned}
$$

where $Y^{\prime}=Y_{5}+Y_{6}$. As we wrote earlier $Y_{5}=\varpi_{1} \rho_{u} a_{3} X_{3}, Y_{6}=\varpi_{1} \rho_{u} a_{4} X_{4}$. In addition, $Y_{5}, Y_{6}$ are independent exponentially distributed random variables with means $\lambda_{5}=1 /\left(\varpi_{1} \rho_{u} a_{3} \delta_{3}^{2}\right)$, $\lambda_{6}=1 /\left(\varpi_{1} \rho_{u} a_{4} \delta_{4}^{2}\right), \beta_{t}=\frac{\gamma_{\text {thf }} a_{2}}{a_{1} \delta_{1}^{2}}+\frac{1}{\delta_{2}^{2}}$.

$f_{4}^{\prime}$ and $f_{5}^{\prime}$ are easily obtained as follows 


$$
\begin{aligned}
& f_{4}^{\prime}=\operatorname{Pr}\left(\frac{\rho_{r}\left|h_{3}\right|^{2} b_{2}}{\rho_{r}\left|h_{3}\right|^{2} b_{1}+\varpi_{2} \rho_{r}\left|h_{3}\right|^{2}+1}>\gamma_{t h m}\right) \\
& =\operatorname{Pr}\left(X_{3}>\tau_{2}^{\prime}\right) \\
& =e^{-\frac{\tau_{2}^{\prime}}{\delta_{3}^{2}}},
\end{aligned}
$$

where $\tau_{2}^{\prime}=\frac{\gamma_{t h m}}{\rho_{r}\left(b_{2}-\gamma_{t h m}\left(b_{1}+\varpi_{2}\right)\right)}$ with $b_{2}>\gamma_{t h m}\left(b_{1}+\varpi_{2}\right)$.

$$
\begin{aligned}
& f_{5}^{\prime}=\operatorname{Pr}\left(\frac{\rho_{r}\left|h_{4}\right|^{2} b_{2}}{\rho_{r}\left|h_{4}\right|^{2} b_{1}+\varpi_{2} \rho_{r}\left|h_{4}\right|^{2}+1}>\gamma_{t h f}\right) \\
& =\operatorname{Pr}\left(X_{4}>\tau_{2}^{\prime}\right) \\
& =e^{-\frac{\tau_{2}^{\prime}}{\delta_{4}^{2}}} .
\end{aligned}
$$

Combining (D.2), (D.3) and (D.4) into (D.1), we can obtain (15).

The proof is completed.

\section{References}

[1] Z. Ding, F. Adachi, and H. V. Poor, "The Application of MIMO to Non-Orthogonal Multiple Access,” IEEE Trans. Wireless Commun., vol. 15, no. 1, pp. 537-552, Jan 2016.

Article (CrossRef Link).

[2] J. Zeng, T. Lv, R. P. Liu, X. Su, M. Peng, C. Wang, and J. Mei, "Investigation on Evolving Single-Carrier NOMA Into Multi-Carrier NOMA in 5G,” IEEE Access, vol. 6, pp. 48268-48288, 2018. Article (CrossRef Link).

[3] J. An, K. Yang, J. Wu, N. Ye, S. Guo and Z. Liao, "Achieving Sustainable Ultra-Dense Heterogeneous Networks for 5G," IEEE Commun. Mag., vol. 55, no. 12, pp. 84-90, Dec. 2017. Article (CrossRef Link).

[4] S. M. R. Islam, N. Avazov, O. A. Dobre, and K. Kwak, "Power-Domain Non-Orthogonal Multiple Access (NOMA) in 5G Systems: Potentials and Challenges," IEEE Communications Surveys Tutorials, vol. 19, no. 2, pp. 721-742, Secondquarter 2017. Article (CrossRef Link).

[5] D. Duchemin, J. Gorce, and C. Goursaud, "Code Domain Non Orthogonal Multiple Access versus ALOHA: A simulation based study,” in Proc. of 2018 25th ICT, pp. 445-450, Jun. 2018.

Article (CrossRef Link).

[6] F. A. Rabee, K. Davaslioglu, and R. Gitlin, "The optimum received power levels of uplink non-orthogonal multiple access (NOMA) signals," in Proc. of 2017 IEEE 18th WAMICON, pp. 1-4, Apr. 2017. Article (CrossRef Link).

[7] B. Xia, J. Wang, K. Xiao, Y. Gao, Y. Yao, and S. Ma, "Outage Performance Analysis for the Advanced SIC Receiver in Wireless NOMA Systems,” IEEE Trans. Veh. Technol, vol. 67, no. 7, pp. 6711-6715, Jul. 2018. Article (CrossRef Link).

[8] M. Sami, N. K. Noordin, M. Khabazian, F. Hashim, and S. Subramaniam, "A Survey and Taxonomy on Medium Access Control Strategies for Cooperative Communication in Wireless Networks: Research Issues and Challenges," IEEE Commun. Surveys Tutorials, vol. 18, no. 4, pp. 2493-2521, Fourthquarter 2016. Article (CrossRef Link).

[9] T. Cui, F. Gao, T. Ho, and A. Nallanathan, "Distributed Space-Time Coding for Two-Way Wireless Relay Networks," IEEE Trans. Signal Process., vol. 57, no. 2, pp. 658-671, Feb. 2009. Article (CrossRef Link). 
[10] K. Yang, N. Yang, C. Xing and J. Wu, "Relay Antenna Selection in MIMO Two-Way Relay Networks Over Nakagami-m Fading Channels," IEEE Tran. Veh. Technol., vol. 63, no. 5, pp. 2349-2362, Jun 2014. Article (CrossRef Link).

[11] D. E. Simmons and J. P. Coon, "Two-Way OFDM-Based Nonlinear Amplify-and-Forward Relay Systems,” IEEE Trans. Vehicular Technology, vol. 65, no. 5, pp. 3808-3812, May 2016. Article (CrossRef Link).

[12] Y. Qi and X. Wang, "Performance Optimization of Two-Way AF Relaying in Asymmetric Fading Channels,” KSII Trans. Internet \& Information Systems, vol. 8, no. 12, pp. 4432-4450, 2014. Article (CrossRef Link).

[13] X. Chen, L. Guo, C. Dong, J. Lin, X. Li and Charles C. Cavalcante, "Probabilistic Constrained Approach for Distributed Robust Beamforming Design in Cognitive Two-way Relay Networks,” KSII Trans. Internet \& Information Systems, vol. 12, no. 1, pp. 21-40, 2018. Article (CrossRef Link).

[14] G. Li, Y. Wang and P. Zhang, "Optimal Linear MMSE Beamforming for Two Way Multi-Antenna Relay Systems,” IEEE Commun. Lett., vol. 15, no. 5, pp. 533-535, 2011. Article (CrossRef Link).

[15] X. Li, Michail Matthaiou, Y. Liu, Hien Quoc Ngo and L. Li, "Multi-Pair Two-Way Massive MIMO Relaying with Hardware Impairments over Rician Fading Channels,” in Proc. of IEEE Globecom, Abu Dhabi, Dec. 2018. Article (CrossRef Link).

[16] X. Yue, Y. Liu, S. Kang, A. Nallanathan, and Y. Chen, "Modeling and analysis of two-way relay non-orthogonal multiple access systems,” IEEE Trans. Commun., vol. 66, no. 9, pp. 3784-3796, Sep. 2018. Article (CrossRef Link).

[17] X. Wang, M. Jia, I. W. Ho, Q. Guo, and F. C. M. Lau, "Exploiting full-duplex two-way relay cooperative non-orthogonal multiple access,” IEEE Trans. Commun.,vol. 67, no. 4, pp. 2716-2729, 2019. Article (CrossRef Link).

[18] B. Zheng, X. Wang, M. Wen, and F. Chen, "NOMA-Based Multi-Pair Two-Way Relay Networks With Rate Splitting and Group Decoding,” IEEE J. Selected Areas in Commun., vol. 35, no. 10, pp. 2328-2341, Oct. 2017. Article (CrossRef Link).

[19] B. Zheng, M. Wen, C. Wang, X. Wang, F. Chen, J. Tang, and F. Ji, "Secure NOMA Based Two-Way Relay Networks Using Artificial Noise and Full Duplex,” IEEE J. Selected Areas in Commun., vol. 36, no. 7, pp. 1426-1440, Jul. 2018. Article (CrossRef Link).

[20] E. Bjornson, M. Matthaiou, and M. Debbah, “A New Look at Dual-Hop Relaying: Performance Limits with Hardware Impairments,” IEEE Trans. Commun., vol. 61, no. 11, pp. 4512-4525, Nov. 2013. Article (CrossRef Link).

[21] T. T. Duy, T. Q. Duong, D. B. da Costa, V. N. Q. Bao, and M. Elkashlan, "Proactive Relay Selection With Joint Impact of Hardware Impairment and Co-Channel Interference,” IEEE Trans. Commun., vol. 63, no. 5, pp. 1594-1606, May 2015. Article (CrossRef Link).

[22] X. Li and J. Li and P. Takis Mathiopoulos and D. Zhang and L. Li and J. Jin, "Joint Impact of Hardware Impairments and Imperfect CSI on Cooperative SWIPT NOMA Multi-Relaying Systems," in Proc. of IEEE/CIC International Conference on Commun. China (ICCC), Beijing, China, 2018. Article (CrossRef Link).

[23] T. Schenk, RF imperfections in high-rate wireless systems:impact and digital compensation, Springer Verlag, New York, NY, USA, 2008. Article (CrossRef Link).

[24] X. Li and J. Li and J. Jin and L. Li, "Performance analysis of relaying systems over nakagami-m fading with transceiver hardware impairments,” Journal of Xidian University, vol. 45, no. 3, pp. 135-140, Jun 2018. Article (CrossRef Link).

[25] C. Deng, X. Zhao, D. Zhang, X. Li, J. Li, Cavalcante, Charles, "Performance Analysis of NOMA-based Relaying Networks with Transceiver Hardware Impairments,” KSII Trans.Internet and Information Systems, vol. 12, Feb. 2018. Article (CrossRef Link)

[26] A. Papazafeiropoulos, S. K. Sharma, T. Ratnarajah, and S. Chatzinotas, "Impact of residual additive transceiver hardware impairments on rayleigh-product mimo channels with linear receivers: Exact and asymptotic analyses," IEEE Trans. Commun., vol. 66, no. 1, pp. 105-118, Jan 2018. Article (CrossRef Link). 
[27] X. Li and M. Huang and X. Tian and H. Guo and J. Jin and C. Zhang, "Impact of Hardware Impairments on Large-scale MIMO Systems over Composite RG Fading Channels," AEU-International J. Electron. and Commun., vol. 88, pp. 134-140, 2018. Article (CrossRef Link).

[28] K. Guo, D. Guo, B. Zhang, and Y. Huang, "Outage analysis and impairments allocation of two-way opportunistic relaying network with hardware impairments,” in Proc. of 2016 IEEE/CIC International Conference on Commun. China (ICCC), Jul. 2016, pp. 1-6. Article (CrossRef Link).

[29] X. Li, J. Li, Y. Liu, Z. Ding, and A. Nallanathan, "Outage Performance of Cooperative NOMA Networks with Hardware Impairments,” in Proc. of IEEE Global Communications Conference (GLOBECOM'18), Jul. 2018. Article (CrossRef Link)

[30] M. Matthaiou, A. Papadogiannis, E. Bjornson, and M. Debbah, "Two-Way Relaying Under the Presence of Relay Transceiver Hardware Impairments,” IEEE Commun. Lett., vol. 17, no. 6, pp. 1136-1139, Jun. 2013. Article (CrossRef Link)

[31] F. Ding, H. Wang, S. Zhang, M. Dai, "Impact of Residual Hardware Impairments on Non-Orthogonal Multiple Access Based Amplify-and-Forward Relaying Networks,” IEEE Access, vol. 6, pp. 15117-15131, 2018. Article (CrossRef Link)

[32] M. Medard, "The effect upon channel capacity in wireless communications of perfect and imperfect knowledge of the channel,” IEEE Trans. Inf. Theory, vol. 46, no. 3, pp. 933-946, May 2000. Article (CrossRef Link)

[33] X. Zhang, M. Matthaiou, M. Coldrey, and E. Bjo“rnson, "Impact of Residual Transmit RF Impairments on Training-Based MIMO Systems," IEEE Trans. Commun., vol. 63, no. 8, pp. 2899-2911, Aug. 2015. Article (CrossRef Link)

[34] X. Yue, Y. Liu, S. Kang, and A. Nallanathan, "Performance Analysis of NOMA With Fixed Gain Relaying Over Nakagami-m Fading Channels,” IEEE Access, vol. 5, pp. 5445-5454, 2017. Article (CrossRef Link)

[35] S. Nadarajah, “A Review of Results on Sums of Random Variables,” Acta Apple Math, vol. 103, pp. 131-140, sep. 2008. Article (CrossRef Link) 


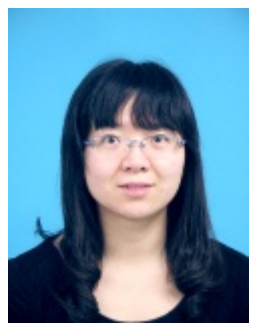

Xinji Tian was born in Henan, china, in 1983. She received Ph.d. degree in signal and information processing from Beijing university of Posts and Telecommunications (BUPT), China in 2011. she is Currently working as Associate professor in School of Physics and Electronic Information Engineering, Henan Polytechnic University. Her research interests include wireless communication, massive MIMO, non-orthogonal multiple access, ultra-dense network.

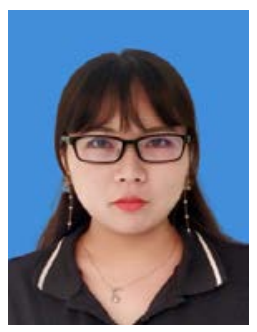

Qianqian Li received the B.Sc degree in communication engineering from the College of Information Engineering, Zhengzhou Shengda University, in 2016. She is currently pursuing the M.Sc. degree in communication and information systems with the School of Physics and Electronic Information Engineering, Henan Polytechnic University, Jiaozuo China. Her current research interests include nonorthogonal multiple access and hardware constrained communication.

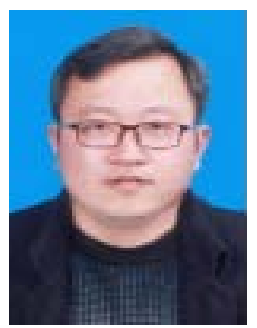

Xingwang Li (S'12-M'15) received his B.Sc. degree in communication engineering from Henan Polytechnic University, China, in 2007. He then received the M. Sc. degree from the National Key Laboratory of Science and Technology on Communications at University of Electronic Science and Technology of China (UESTC), in 2010 and Ph. D. degrees in communication and information system from the State Key Laboratory of Networking and Switching Technology at Beijing University of Posts and Telecommunications (BUPT), in 2015. From 2010 to 2012, he was with Comba Telecom Ltd. in Guangzhou China, as a engineer. He spent one year from 2017 to 2018 as a visiting scholar at the Institute of Electronics, Communications and Information Technology (ECIT), Queen's University Belfast (QUB), Belfast, UK. He is currently Associated Professor with the School of Physics and Electronic Information Engineering, Henan Polytechnic University, Jiaozuo China. He has several papers published in journal and conferences, has authored several patents and he has worked on several funded research projects on the wireless communications areas. His research interests include MIMO communication, cooperative communication, hardware constrained communication, non-orthogonal multiple access, physical layer security, unmanned aerial vehicles, free space optical communications, and performance analysis of fading channels. Dr. Li now serves as an editor of the KSII Transactions on Internet and Information Systems and TPC member of IEEE/CIC ICCC workshop. 19', He also has served a TPC member of IEEE Globecom Workshop. 18'.

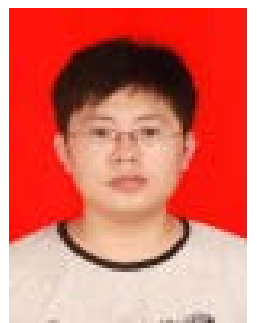

Hui Zhang received his B.Sc. degree in communication engineering from Henan Polytechnic University, China, in 2007. He then received the M. Sc. degree from the School of Energy Science and Engineering, Henan Polytechnic University, in 2010 and Ph. D. degrees from the School of Energy and Mining Engineering, China University of Mining and Technology, in 2013. He is currently a Associated Professor with the School of Energy Science and Engineering, Henan Polytechnic University. He has authored several papers in journal and conferences and holds several patents. His research interests include mining communication and smart mine. 


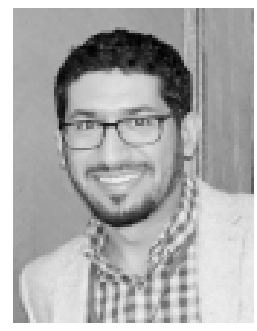

Khaled M. Rabie received the B.Sc. degree (Hons.) in electrical and electronic engineering from the University of Tripoli, Tripoli, Libya, in 2008, and the M.Sc. and Ph.D. degrees in communication engineering from The University of Manchester, Manchester, U.K., in 2010 and 2015, respectively. He is currently a Post-Doctoral Research Associate with Manchester Metropolitan University (MMU), Manchester. His research interests include signal processing and the analysis of power line and wireless communication networks. He was a recipient of the Best Student Paper Award at the IEEE ISPLC, TX, USA, 2015, and the MMU Outstanding Knowledge Exchange Project Award of 2016. He is currently the Program Chair of the IEEE ISPLC 2018, the IEEE CSNDSP 2018 Co-Chair of the Green Communications and Networks track, and the Publicity Chair of the INISCOM 2018. He is also an Associate Editor of the IEEE ACCESS, an Editor of the Physical Communication journal (Elsevier), and a fellow of the U.K. Higher Education Academy.

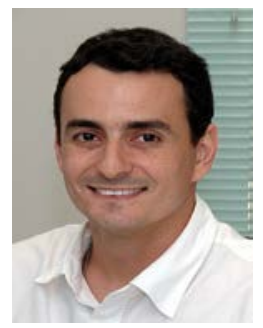

Charles Casimiro Cavalcante received the B.Sc and M.Sc in Electrical Engineering from the Federal University of Cear (UFC), Brazil, in 1999 and 2001, respectively, and the Ph.D. degree from the University of Campinas (UNICAMP), Brazil, in 2004. He has held a grant for Scientific and Technological Development from 2004 to 2007 and since March 2009 he has a grant of Scientific Research Productivity both from the Brazilian Research Council (CNPq). From March 2007 to November 2008 he was a Visiting Professor at Teleinformatics Engineering Department of UFC and since November 2008 he is an Assistant Professor at the same department and university holding the Statistical Signal Processing chair. From August 2014 to July 2015 he was a Visiting Assistant Professor at the Department of Computer Science and Electrical Engineering (CSEE) from University of Maryland, Baltimore County (UMBC) in the United States. He has been working on signal processing strategies for communications where he has several papers published in journal and conferences, has authored three international patents and he has worked on several funded research projects on the signal processing and wireless communications areas. He is also a co-author of the book Unsupervised Signal Processing: Channel Equalization and Source Separation, published by CRC Press. He is a researcher of the Wireless Telecommunications Research Group (GTEL) where he leads research on signal processing and wireless communications. Dr. Cavalcante is a Senior Member of the IEEE and Senior Member of the Brazilian Telecommunications Society (SBrT). His main research interests are in signal processing for communications, blind source separation, wireless communications, and statistical signal processing 